\title{
Analyser des vidéos sur les pratiques des enseignants du second degré en mathématiques : des utilisations contrastées en recherche en didactique et en formation de formateurs - quelle transposition?
}

Using videos analysis as a tool to work on mathematics teachers' practices at secondary education level: similarities and differences between research and educators training

\section{Aline Robert et Laurent Vivier}

\section{OpenEdition}

Journals

Édition électronique

URL : https://journals.openedition.org/educationdidactique/1749

DOI : 10.4000/educationdidactique. 1749

ISSN : 2111-4838

Éditeur

Presses universitaires de Rennes

Édition imprimée

Date de publication : 30 octobre 2013

Pagination : 115-144

ISBN : 978-2-7535-3255-7

ISSN : 1956-3485

\section{Référence électronique}

Aline Robert et Laurent Vivier, « Analyser des vidéos sur les pratiques des enseignants du second degré en mathématiques : des utilisations contrastées en recherche en didactique et en formation de formateurs - quelle transposition? », Éducation et didactique [En ligne], 7-2 | 2013, mis en ligne le 31 octobre 2015, consulté le 25 avril 2022. URL : http://journals.openedition.org/educationdidactique/ 1749 ; DOI : https://doi.org/10.4000/educationdidactique.1749 


\title{
ANALYSER DES VIDÉOS SUR LES PRATIQUES DES ENSEIGNANTS DU SECOND DEGRÉ EN MATHÉMATIQUES: DES UTILISATIONS CONTRASTÉES EN RECHERCHE EN DIDACTIQUE ET EN FORMATION DE FORMATEURS - QUELLE TRANSPOSITION?
}

\author{
Aline Robert, LDAR, université Paris-Diderot, université Cergy-Pontoise
}

Laurent Vivier, LDAR, université Paris-Diderot

\begin{abstract}
Dans cet article nous illustrons les différences d'analyses de vidéo de séances de classe selon qu'elles s'insèrent dans des recherches en didactique ou au cours d'une formation de formateurs. Après avoir éclairé le point de vue théorique adopté, issu de la théorie de l'Activité, commun aux deux contextes, nous présentons en toute généralité les analyses de vidéo dans chacun des environnements. Il s'agit de dégager des éléments constitutifs de la transposition que nous faisons de la recherche vers la formation de formateurs. Nous développons ensuite un exemple précis, basé sur un extrait de vidéo, sur lequel nous illustrons les deux types d'utilisation. En conclusion, nous élargissons la réflexion à des questions et aux perspectives, en insistant sur le rôle clef que peuvent jouer les formateurs.
\end{abstract}

Mots-clés: didactique de l'histoire, modèle disciplinaire, jeux d'apprentissage, conceptualisation historique, théorie de l'action conjointe en didactique.

Using videos analysis as a tool to work on mathematics teachers' practices at secondary education level: similarities and differences between research and educators training

In this article we begin by describing the theoretical framework on which rely our videos analyses both in research and in educators' training. This framework is inspired by the Activity theory, as referring to "the activity of a subject within a specific situation". Then we describe our videos analysis of pieces of classrooms' lessons. Actually we are mainly concerned here with the research tools which are used for the educators training, and with the adaptation of their uses according to the context: it is what we call the transposition of these tools. To enhance the reader's understanding, we develop a specific case on which we show both analyses. In the conclusion we insist on the importance of the educators 'work, and on the challenge of their specific training.

Keywords: videos analysis, educators training, mathematics education, analysis of mathematical tasks, mathematics teachers' practices, transposition of research tools. 


\section{INTRODUCTION}

Que ce soit dans les recherches sur les pratiques des enseignants de mathématiques ou les formations de ceux-ci, l'utilisation de vidéos tournées pendant des séances de classe s'est généralisée, grâce au perfectionnement du matériel notamment - caméscopes numériques très maniables, numérisation des vidéos et mise sur ordinateur très simple... Nous avons développé depuis une quinzaine d'années plusieurs utilisations contrastées de ce type de matériel, avec toutefois deux points communs : nous travaillons dans tous les cas (jusqu'à présent) à partir de vidéos tournées en classe, caméscope posé $^{1}$ au fond de la salle, centré sur le tableau, avec comme acteur principal l'enseignant - ces choix seront discutés en conclusion. Le cadre théorique qui légitime et oriente ces recherches et ces formations qui mettent en questionnement les pratiques des enseignants, est le même - celui de la double approche ergonomique et didactique des pratiques, inscrite dans la théorie de l'Activité (Robert \& Rogalski, 2002, 2005, Rogalski, 2008, Vandebrouck, 2008). L'activité est ce que développe un sujet lors de la réalisation d'une tâche en situation - ses actes extériorisés mais aussi les hypothèses, les décisions (ce qu'il pense) dans ce qu'il fait (dit) ou non - cela comprend son « état personnel ». L'activité effective est, par définition, inaccessible mais laisse des traces observables. Ce cadre unificateur permet d'aborder les activités des élèves et de l'enseignant et de préciser les hypothèses en formation de formateurs à l'origine des analyses adoptées en formation. Il y a une proximité certaine avec la démarche de Goigoux pour le français et les Professeurs des Écoles (2007), même si l'exploitation que fait ce dernier de la description du travail de Leplat (1997) est plus importante que dans nos travaux, notamment en termes de distinction des tâches prescrites et redéfinies.

Le mot "pratiques » désigne ici très généralement ce que les enseignants pensent, disent et ne disent pas, font et ne font pas, en classe et hors de la classe. Cela comprend les activités en situation, et notamment le travail de l'enseignant pour la classe et en classe, qui nous intéresse tout particulièrement ici. Ce travail, constitué d'activités précises, notamment en classe, n'est donc qu'une partie des pratiques que développent les enseignants.

En ce qui concerne la formation, pour ne pas généraliser trop le propos, nous nous sommes limités dans ce texte à décrire l'utilisation de vidéos faite dans la formation de formateurs proposée dans le cadre d'un master professionnel ouvert à l'université Paris Diderot (il ne s'agit donc pas d'une formation d'enseignants, cela doit être souligné, même si les participants sont des enseignants ayant exercé depuis plus de 5 ans). L'objectif de cette formation est de donner aux futurs formateurs les moyens d'assurer cette place bien particulière entre les enseignants, l'institution et les chercheurs, presque entre l'enclume et le marteau. Ils ont besoin d'outils à la fois pour apprécier les injonctions institutionnelles et les recherches, pour s'adapter aux enseignants qu'ils connaissent bien mais en se décentrant de leurs propres choix d'enseignant, en ouvrant la palette des possibles pour tous, et pour disposer de quelques leviers concernant les formations professionnelles adaptées au travail spécifique des enseignants. Enfin ils ont un rôle important à jouer dans la nécessaire liaison entre les enseignants et les chercheurs, et cela ne s'improvise pas! Même s'il n'y a pas de recherche sur cette formation de formateurs ${ }^{2}$, pas d'évaluation, la question des outils d'analyse des séances de classe, développés pour les analyses de vidéos, et transposés de la recherche sur les pratiques aux formateurs nous semble importante, nous en discuterons après les avoir décrits. Nous préciserons aussi l'accès aux pratiques que nous déléguons au visionnement des vidéos tel qu'il est prévu, dans chaque dispositif, et nos attentes correspondantes en matière de formation.

Cela sera facilité par le fait que cette formation de formateurs est mise en place par un (chercheur)formateur depuis plus de 10 ans et décrite ici par deux chercheurs-(formateurs), dont l'initiateur.

Dans cet article nous allons éclairer rapidement et synthétiquement le point de vue théorique adopté tant dans nos recherches que pour notre formation de formateurs. Puis, nous présentons en toute généralité les analyses de vidéo dans les deux environnements, du point de vue méthodologique. Le point de vue de la recherche, rapidement évoqué, sert surtout à mettre en valeur les différences entre les deux utilisations. Nous développons ensuite un exemple précis sur lequel nous illustrons les deux types d'utilisation d'un extrait de vidéo. Il est à noter que le point de vue de la formation de formateurs est plus développé puisque les analyses de vidéo y sont centrales. L'exercice est un peu artificiel dans la mesure où une partie des dispositifs ne peut être 
restituée. En particulier, nous nous limitons aux utilisations de la vidéo sans chercher à évaluer les dispositifs correspondants. Nous apprécierons les limites de notre étude en conclusion, en présentant aussi des perspectives dans les deux directions et en élargissant la réflexion aux formateurs.

\section{NOS PARTIS PRIS THÉORIQUES POUR QUESTIONNER LES PRATIQUES DES ENSEIGNANTS À PARTIR D'ANALYSES DE VIDÉO}

Pour les mathématiques, questionner les pratiques des enseignants se décline en plusieurs interrogations dont les suivantes, naïves :

- Quels types de liens retient-on entre les pratiques des enseignants et les apprentissages mathématiques des élèves?

- Qu'est-ce qui peut varier dans les pratiques enseignantes individuelles, collectives ?

- Comment les pratiques se développent-elles - au début et tout au long de la carrière?

Le premier questionnement, dont on connaît depuis fort longtemps la complexité au vu des paramètres engagés et de la difficulté à cerner « les apprentissages », est renseigné de manière renouvelée dans notre cadre théorique par un certain nombre de travaux didactiques qui mettent en relation des productions d'élèves et des pratiques effectives d'enseignant $^{3}$ (de mathématiques). L'étude des marges de manœuvre des enseignants amène à préciser les choix individuels, entre des questions locales, qui intéressent une séance donnée, et des découpages plus globaux, qui intéressent toute une catégorie d'enseignants et qui sont très liés aux programmes et aux horaires autorisés - autant de contraintes partagées. Enfin les projets de formation élaborés par les chercheurs, à expérimenter, mettent en jeu des hypothèses sur le développement des pratiques, à tester. Nous précisons ci-dessous notre démarche théorique pour aborder ces questions, en présentant nos « partis pris".

Questionner les pratiques d'enseignants, en lien avec les activités des élèves et le métier - un point de vue théorique

Comme cela a été annoncé, toutes nos recherches s'inscrivent dans la théorie générale de l'Activité, spécifiée aux mathématiques et aux situations scolaires. Les apprentissages des élèves à chaque niveau sont ainsi d'une part référés aux niveaux de conceptualisation visés, eux-mêmes définis par les chercheurs à partir des programmes et des spécificités des notions. Cette référence à la conceptualisation est un premier parti pris théorique. Il nous amène à adapter aux mathématiques et aux situations scolaires des théories de l'apprentissage et des éléments épistémologiques et didactiques, en tenant compte des difficultés des élèves qui ne résultent pas toujours directement de la seule structure conceptuelle $^{4}$ (cf. Vergnaud, 1991). Il s'agit par exemple de dégager les objets et les outils mathématiques dont on veut construire la disponibilité ${ }^{5}$ qui inclut la nécessaire (ré)organisation des connaissances qui doit se faire. Pour cela, on associe à ces objets et outils un ensemble de tâches mathématiques susceptibles de provoquer cette disponibilité tout en permettant de la tester, le choix et l'ordre de ces tâches tenant compte des mathématiques et des difficultés répertoriées.

D'autre part, et cela constitue notre deuxième parti pris théorique, ces apprentissages visés sont étudiés à partir de l'enseignement dispensé et des activités possibles correspondantes des élèves, en donnant ainsi toute leur place aux sujets en situation scolaire - même si cela reste partiel pour comprendre tout ce qui se joue ${ }^{6}$. Il manque notamment tout ce qui peut jouer hors-classe mais aussi des aspects affectifs et sociaux, dont nous reconnaissons cependant l'importance. Les activités sont étudiées surtout en classe. Dans la mesure où elles sont en partie inaccessibles (cf. ce que pensent les élèves), nous dégageons les activités « possibles », inférées par le chercheur à partir du croisement des tâches proposées et de ce que donne à voir et à entendre le déroulement. L'adjectif « possibles » souligne la différence entre ce que propose l'enseignant, l'observable, et ce qui va en résulter pour les élèves, l'effectif. Le fait que la vidéo est centrée sur l'enseignant prive certainement de certains éléments qui pourraient apparaître si on filmait les élèves, voire leurs cahiers, mais comment filmer tous les élèves? Le compromis choisi, partir de l'enseignant pour décoder ce qui s'est passé, correspond au fait que nous nous intéressons à ce qui peut dépendre de l'enseignant, justement.

Outre le fait que, par définition même, les activités des élèves et des enseignants sont en partie inaccessibles, il y a une autre difficulté méthodologique immédiate, permanente, sur la définition et la 
taille de ces activités. En général nous les calibrons en relation avec les tâches à accomplir et les différentes connaissances à mettre en œuvre côté élèves, l'analyse des activités des enseignants étant pilotée par celles des élèves.

Ainsi, de notre point de vue, ce qui est important est que les activités des élèves sont en grande partie provoquées par celles de l'enseignant, c'est du moins ce à quoi nous pouvons accéder : le troisième parti pris théorique est de décoder les activités de l'enseignant à partir de celles supposées des élèves, et réciproquement, en repérant notamment d'éventuels décalages ou renforcements.

Seulement nos premières recherches sur les pratiques, exclusivement reliées à ces seuls apprentissages, restaient peu interprétables (Robert, 2010). L'ajout de la référence à la didactique professionnelle et à la psychologie ergonomique nous a amenés à penser que la prise en compte de ce seul aspect, celui des apprentissages visés, est insuffisant pour comprendre ce qui se joue dans les choix des enseignants ${ }^{7}$. Nous rapportons ainsi les pratiques observées à l'exercice de leur métier par les enseignants, en tenant compte explicitement de leur inscription professionnelle et personnelle qui peut avoir des incidences sur la classe et les activités des élèves. En particulier, les choix de contenus mathématiques que fait un enseignant sont certes impliqués par la nature même des mathématiques en jeu, mais aussi par des impératifs de gestion de la classe concernée, par des considérations liées aux programmes, au temps long, à ses propres représentations et connaissances.

Comme nous avons eu l'occasion de le développer (cf. double approche didactique et ergonomique, Robert et Rogalski 2002, 2005, Masselot et Robert, 2007, Robert, 2008), ce travail professionnel des enseignants en classe et pour la classe est complexe : il se joue à plusieurs niveaux de temporalité, donnant lieu à des analyses selon plusieurs granularités et résulte d'une recomposition de différents objectifs qui ne sont pas tous liés aux seuls élèves, il se déroule en plusieurs phases distinctes à la fois indépendantes et dépendantes, il met en jeu de manière très imbriquée des contraintes de différentes natures - institutionnelles, sociales, personnelles.
Les analyses de vidéo en recherche - un outil pour accéder aux activités mathématiques des élèves et des enseignants en classe

C'est le cadrage théorique qui vient d'être esquissé qui justifie l'utilisation des vidéos en recherches pour donner accès à ce qui se joue en classe $e^{8}$ - cette utilisation sera décrite plus précisément en section II. Il s'agit en particulier de renseigner la question suivante : comment les vidéos donnent-elles accès aux pratiques dans notre point de vue?

En réalité, ce que permettent de renseigner les vidéos et qui nous intéresse, ce sont les choix, observables ou inférables à partir des données, des enseignants en classe. En effet, à partir de leur préparation, les enseignants s'adaptent en improvisant plus ou moins, pour provoquer les activités attendues des élèves. Ces choix font partie de l'intelligibilité des phénomènes d'enseignement et d'apprentissage que nous traquons.

\section{Des pratiques enseignantes aux activités des élèves}

Nous analysons les pratiques des enseignants d'une part pour mieux comprendre les activités possibles des élèves, trop mal définies à partir des seules tâches mathématiques proposées, même si on s'intéresse à la totalité des tâches sur un chapitre. Nous pensons en effet que les analyses de vidéos peuvent contribuer à la compréhension du lien pratiques enseignantes/activités des élèves ${ }^{9}$. Pour cela, ce sont les déroulements des séances que nous étudions à l'aide de vidéos et de compléments indispensables, notamment sur les contextes - la classe, l'établissement, l'inscription des séances dans l'année - et l'ensemble des tâches proposées (reconstituées ou fournies par l'enseignant). C'est en croisant les tâches proposées aux élèves avec le travail des élèves qu'organise l'enseignant sur ces tâches qu'on accorde aux activités des élèves l'importance qu'on leur reconnaît dans la théorie de l'Activité, en relation avec les apprentissages. La recherche d'activités possibles « a minima » et " a maxima ${ }^{10}$ est un moyen pour appréhender l'hétérogénéité des élèves pendant un déroulement. Cependant, une difficulté constante tient à la définition des observables, à leur renseignement et surtout à leur recomposition en activités distinctes différentes pour chaque élève. 
Rappelons que, par définition, ces activités sont en partie hors d'atteinte et que l'on cherche à les cerner par les activités possibles déduites de l'observation. Une deuxième difficulté constante tient à l'appréciation de l'ensemble de ces activités, nécessaire pour appréhender ce qui peut contribuer aux apprentissages, c'est-à-dire à l'imbrication du niveau d'observation local, détaillé, dans ce que peuvent révéler des analyses globales.

\section{Pratiques enseignantes}

D'autre part, ces analyses de séances par l'intermédiaire de vidéos servent à améliorer notre intelligibilité des pratiques enseignantes, à travers les régularités et diversités intra et inter-individuelles. Cela amène à élaborer un état des marges de manœuvre, des contraintes et d'autres facteurs de variabilités des pratiques, qui rejaillissent sur les activités des élèves. Nos recherches sur les pratiques des enseignants ont ainsi permis d'approfondir leur complexité. La méthodologie, issue de la double approche, amène en particulier à reconstituer des éléments non observables, « manquants », et à passer du quotidien (local) à un niveau plus global prenant en compte les régularités et les stabilités individuelles - les logiques d'action. C'est le croisement systématique des cinq composantes introduites dans la double approche avec l'introduction des différents niveaux d'organisation des pratiques (micro, local, global) qui autorisent ces incursions dans la complexité à partir des analyses de classes filmées (Robert, 2008). Les composantes cognitive et médiative traduisent les choix de contenus et de gestion, elles reprennent ce qui est observable à partir des séances de classe ; les composantes institutionnelle, sociale et personnelle permettent de les compléter par des déterminants liés aux personnes et au métier, en relation avec les contraintes institutionnelles, notamment les programmes et les horaires, et sociales, liées aux compositions des classes et aux établissements notamment. Les niveaux d'organisation permettent d'accéder systématiquement et de manière non indépendante aux projets de l'enseignant (niveau global), à ses routines et automatismes (micro), et à son travail au quotidien, en classe (niveau local), qui convoque à la fois les préparations et des improvisations, facilitées par le recours à des automatismes adaptés et par la vision globale qu'a l'enseignant de ses objectifs.
On a pu montrer en utilisant ce cadrage (cf. par exemple les travaux de Roditi, 2003, Horoks, 2008, Chesnais, 2009) que les régularités inter-individuelles sont formatées en bonne partie par les contraintes des programmes et des horaires, des établissements, alors que les diversités, liées aux investissements différenciés des marges de manœuvre, plus locales, sont plus attachées à des choix de contenus (circonscrits cependant par un champ scientifique commun) et de gestion - renvoyant à ce qui les gouverne en termes de représentations, d'expériences et de connaissances. La gestion qu'un enseignant adopte n'est pas toujours vue par lui comme résultant d'un choix, mais plutôt comme un équilibre « optimal » - c'est le chercheur qui la présente ainsi, dans sa démarche de traques d'alternatives. Enfin, la stabilité des pratiques des enseignants en régime de croisière, constatée dans nos travaux après avoir été déjà supposée par d'autres (Crahay, 1989), semble plus liée à ces " choix » de déroulement (composantes médiative et personnelle) qu'aux choix de contenus, plus faciles et moins coûteux à faire changer (Robert, 2007, Chappet-Paries, Robert et Rogalski, 2008).

Enfin les débutants manifestent une « surcharge du niveau local », cette expression faisant référence à la pauvreté de leurs automatismes, pas encore construits au niveau micro, et, souvent de leurs projets globaux, encore parcellaires. Leurs choix sont ainsi souvent caricaturaux, ou bien ils laissent de côté les élèves - majorant leur projet mathématique pour la séance donnée - ou au contraire ils se laissent déborder par les élèves, au détriment du projet d'enseignement (Chesné, 2006).

\section{Les analyses de vidéo en formation d'enseignants et de formateurs - un outil pour travailler les pratiques}

Tout ce qui précède intervient en formation. Nous nous inspirons encore du cadre de la théorie de l'Activité pour réfléchir aux formations, notamment pour éclaircir notre position sur la question suivante : comment les vidéos peuvent-elles contribuer à former des enseignants, des formateurs ?

Les hypothèses que nous adoptons sur le développement des pratiques enseignantes constituent notre dernier parti pris théorique et éclairent la question de l'utilisation des vidéos en formation (qui s'inscrit bien entendu dans un dispositif plus large, nous y 
reviendrons). Nous avons adopté un modèle inspiré de la Zone Proximale de Développement (Vygotski, 1997) adapté aux pratiques (Valsiner, 1992, Robert, 2010) - avec toutes les conséquences correspondantes sur les modalités de ces formations. Un slogan (avec les défauts de tout slogan) pourrait résumer cette hypothèse : " partir des pratiques (presque) déjà-là pour les former ».

\section{Formation d'enseignants ${ }^{11}$}

Dans cette démarche, le travail en formation d'enseignants s'appuie sur la tentative de faire adopter d'emblée aux participants une posture d'enseignant, à partir d'une « vraie » séance de classe, avec les besoins ressentis correspondants, exprimés, multidimensionnels, véritable point de départ de ce qui va se jouer. Les études de vidéos contribuent à amorcer le travail souhaité, en permettant l'adoption de cette posture et la discussion à partir de cette posture. C'est le formateur qui, ayant pris connaissance de l'« état des questionnements », doit rapprocher ces derniers des besoins supposés (par lui), petit à petit, en instruisant le dossier enseignant le plus complet possible, en provoquant des prises de conscience, en présentant des compléments, des alternatives et en remontant à des questions plus générales ensuite. Les besoins supposés que nous retenons sont relatifs aux choix qu'ont à faire des enseignants, tant sur l'élaboration des scénarios ${ }^{12}$ sur un chapitre (faisant intervenir un projet global et le niveau local par des choix de tâches) qu'en matière de déroulement des séances et de gestion en classe du travail des élèves. L'ambition est d'enrichir la palette des possibles des enseignants, en leur donnant des moyens d'accéder à divers choix et de tenir compte des diverses contraintes. Le départ contextualisé dans la classe, notamment grâce aux vidéos, le visionnement collectif et discuté, facilitent les compréhensions mutuelles et les appropriations, à partir de prises de conscience, éclairées par la discussion, et de présentation d'alternatives précises, en contexte $^{13}$, argumentées.

De fait, nos hypothèses sur le développement des pratiques nous amènent à concevoir, pour les enseignants, des formations «à l'envers ». On commence par l'analyse de séances et du travail correspondant de l'enseignant (notamment par l'intermédiaire de vidéos). De ce travail préliminaire au niveau local, sur une seule séance ou sur un extrait, où sont étudiés les tâches, les déroulements, les contraintes et les alternatives, découlent les interventions plus générales sur les mathématiques à enseigner, les programmes, etc. Même si selon le type de formation, initiale ou continue notamment, les choix précis diffèrent, il s'agit de prendre comme point de départ du processus de formation professionnel, à un moment donné, les besoins ressentis par les enseignants lorsqu'ils sont en classe. Ces besoins sont liés aux déroulements et aux objectifs poursuivis à partir de la préparation, faisant intervenir la fin de la démarche de l'enseignant, qui a d'abord mis au point ses séances, en réfléchissant à la notion visée et aux programmes. Cette inversion temporelle, le fait de partir de la fin, de ce qui se passe en classe alors qu'un travail préalable a déjà eu lieu, hors-classe, est à l'origine de cette dénomination « à l'envers » que nous avons proposée.

Ce choix de formation est lié à la définition des pratiques que nous adoptons et aux besoins spécifiques, liés au métier et à la classe et pas seulement aux apprentissages et aux choix de contenus, d'ailleurs déjà un peu préparés par les études antérieures ; il est adapté à nos hypothèses sur les formations professionnelles, postulant l'intérêt de faire travailler les formés en leur faisant adopter une posture proche de la posture professionnelle visée. Cela permet l'expression des besoins correspondants et leur rapprochement avec les formations, les formateurs étant en mesure, grâce à ce type de dispositifs, d'adapter leurs propos. C'est le travail de transposition correspondant, qui permet de tenir compte de ce qui est exprimé par les enseignants en formation et de s'appuyer dessus pour élargir le débat qui est spécifique de la formation des formateurs.

\section{Formation de formateurs}

En formation de formateurs, la même démarche initiale est adoptée, suivant une forme d'homologie, faisant vivre aux futurs formateurs ce qu'ils auront à animer plus tard eux-mêmes (cf. Kuzniak, 1994). Le double travail explicite sur les marges de manœuvre de chacun et sur les contraintes incontournables, notamment liées aux mathématiques à enseigner et aux élèves, est évidemment un élément clef de ce dossier. En lien avec la double approche, les raisons des acteurs sont prises en compte ainsi que la visée d'enrichissement grâce à l'explicitation de la palette des possibles sur un contenu donné. 
Nos recherches sur les pratiques et notre utilisation de vidéos en recherche nous amènent à conserver l'idée, pour la formation des formateurs, de travailler sur les séances de classe comme révélateur essentiel d'une partie du travail de l'enseignant, en lien avec sa préparation (analyses des tâches au sein d'un scénario), le déroulement qu'il organise, les contraintes qui pèsent sur la séance - liées aux élèves, aux mathématiques, à l'institution, mais aussi personnelles... Chaque vidéo est étudiée systématiquement, pour que suffisamment d'exemples aient été travaillés pour préparer ce qui sera rencontré ensuite.

Ainsi l'enjeu de la formation de formateurs est-il de donner aux futurs formateurs des moyens pour, dans un premier temps, apprécier à la fois les qualités des scénarios globaux proposés par les enseignants, même s'ils sont différents de ceux qu'ils auraient eux-mêmes choisis, et la proximité ou les décalages pendant une séance entre ce qui est attendu (analysé a priori) et ce qui se passe, en abordant des causes possibles - c'est ainsi la richesse de l'ensemble des tâches et leur robustesse qui sont questionnées, c'est-à-dire leur potentiel de déformation pendant les déroulements (plus une tâche est robuste, moins elle est susceptible d'être déformée). Dans un deuxième temps, ce sont les moyens pour concevoir des formations « adaptées » à nos hypothèses sur le développement des pratiques qui sont visés. Cela illustre ce que, en tant que formateur de formateurs, nous appelons les besoins supposés des formateurs pour l'élaboration de séances de formation à organiser par eux dans le futur.

Notre position donne une importance considérable aux formateurs (nous y reviendrons en conclusion) et se distingue de celle des chercheurs s'intéressant aux formations qui se polarisent davantage sur les différents types de savoirs dont les enseignants auront besoin (point de vue largement adopté dans le monde anglo-saxon, Shulmann, 1986, Ball et Bass, 2003).

\section{Premier aperçu de la transposition recherche $\rightarrow$ formation de formateurs}

On peut donner un premier aperçu, qui sera illustré et discuté plus loin, de ce que les chercheurs transposent (notamment des analyses de vidéo) lorsqu'ils deviennent formateurs de formateurs (notamment lors des analyses de vidéos). Cependant plusieurs niveaux interviennent de manière imbriquée, qui ne concernent pas tous les analyses de vidéos, qui sont, de ce fait, difficiles d'isoler complètement dans cet effort de clarification.

En formation de formateurs, on admet l'intérêt d'outiller l'expérience déjà acquise des futurs formateurs par un travail, systématique et progressif, sur des variables afférentes aux activités mathématiques des élèves et au travail de l'enseignant, directement issues de recherches en didactique. Ces variables, que les futurs formateurs doivent s'approprier, sont présentées comme pouvant « influencer les activités mathématiques des élèves », en étant susceptibles de donner lieu à des choix différents des enseignants. Ainsi transpose-t-on des outils (méthodologiques pour le chercheur), permettant de faire des analyses de tâches et des analyses de déroulement de séances. Cela prend souvent la forme de listes de variables à renseigner qui sont données après un travail en séance sur des exemples (exercices puis vidéos à analyser, ou étude d'un domaine des mathématiques) - ces variables apparaissent dans l'étude des exemples ci-dessous. Cependant les justifications théoriques correspondantes ne sont pas développées, seulement esquissées dans une séance «théorique ». Ces variables sont donc décontextualisées, à partir de la démarche complète leur donnant sens, et prennent une importance différente, elles deviennent plus « autonomes ${ }^{14}$.

Ce qui doit être souligné, c'est cette idée issue du cadre théorique adopté et en quelque sorte " imposée » en formation de formateurs, à savoir que les choix faits par chaque enseignant pour ces variables ne sont pas indépendants, il y a des relations dans tous les sens à élucider, en référence à la complexité des pratiques. Et ceci vaut aussi bien pour le chercheur qui cherche à comprendre des pratiques que pour le formateur qui élabore un dispositif de formation ou encore un enseignant qui construit son cours. Par exemple, les mathématiques à enseigner, au programme, entrainent des choix de contenus, mais les contraintes de gestion de la classe influencent aussi ces choix, peut-être autrement. De même, pour les apprentissages des élèves on fait adopter l'idée que ce sont les activités qui sont centrales et qui permettent de renseigner, partiellement, les apprentissages et les pratiques.

L'objectif explicite est un enrichissement de la palette des choix possibles à faire adopter dans leur 
travail par les enseignants à former par la suite. Cet enrichissement visé par les formations qui seront organisées par les futurs formateurs (qui concernent l'installation dans le métier ou la suite) ne résulte pas de la transmission directe des variables en question. Il s'agit plutôt d'élaborer un dispositif de formations « à l'envers » dans lequel le formateur dispose de ces variables et s'appuie dessus, sans nécessairement les expliciter, pour mettre en valeur ce qu'il a décidé de travailler. Ainsi, le formateur d'enseignants aura, lui aussi, à effectuer une nouvelle transposition.

On travaille aussi sur les mathématiques à enseigner (ce que nous appelons le relief, voir ci-dessous), voire les scénarios, avec des exemples (non exhaustifs) qui font l'objet de synthèses originales du formateur ${ }^{15}$, faites à partir de recherches mais n'en reprenant pas toujours tout. Il y a là des éléments transposés à apprendre par les participants.

Reste la dernière forme de transposition qui concerne les modalités des formations : le schéma des « formations à l'envers », amorcées par l'étude du local, du travail en classe, abordé dans sa complexité, qui est adopté en formation de formateurs, est issu d'hypothèses théoriques admises en recherche; il est ensuite commenté, argumenté, et proposé pour organiser aussi les formations d'enseignants. Ce schéma est directement issu des hypothèses que nous développons en recherche à partir de nos résultats sur les analyses de pratiques et de notre cadre théorique, il représente une manière de contextualiser nos hypothèses, en mettant en jeu des vidéos pour entrer en classe. Rappelons qu'il n'y a pas encore de recherche sur les effets de ce type de formations, que ce soit pour les formateurs ou les enseignants.

\section{DESCRIPTION DES UTILISATIONS DE VIDÉOS EN RECHERCHE ET EN FORMATION DE FORMATEURS}

Dans cette section, nous décrivons rapidement et en toute généralité les deux types d'utilisation de vidéos envisagés dans cet article afin de préciser le contexte général de notre propos. Ces analyses sont apparues dans nos travaux d'abord en recherche, même si l'usage en était déjà introduit depuis longtemps en formation, par exemple par le biais du micro-enseignement (Altet et Donaro, 1985).

\section{Analyses de vidéos en recherche}

Les analyses de vidéo que nous utilisons dans certaines recherches sur les pratiques des enseignants s'inscrivent comme une étape d'une démarche en plusieurs actes, inscrite dans le cadre théorique commun, décrit dans la première partie. Nous en donnons un canevas général, en soulignant que ces recherches mettent en jeu une problématique précise, variable selon les travaux, avec une méthodologie qui doit être adaptée à chaque fois. Il s'agit de permettre de repérer la transposition entre ces recherches et ce qui est adopté en formation de formateurs.

À titre d'exemple, et pour nous limiter à des travaux récents proches des nôtres, Roditi (2003) a ainsi étudié les régularités et les diversités des pratiques en classe d'enseignants sur un même contenu et à un même niveau scolaire (la multiplication des décimaux en sixième) et dans des établissements comparables (ordinaires), à partir de vidéos filmées dans les classes des différents enseignants étudiés. Dans d'autres travaux, Horoks $(2008)^{16}$ a mis en relation des pratiques en classe de plusieurs enseignants d'un même niveau scolaire sur un même chapitre du programme, toujours analysées à partir de vidéos tournées en classe, et des productions correspondantes des élèves, sans faire intervenir de différences sociales entre établissements Une comparaison faisant intervenir la composition sociale des classes a été réalisée sur un même chapitre dans deux classes de sixième, là encore en s'appuyant sur des vidéos tournées en classe (Chesnais, 2009) ${ }^{17}$.

\section{Avant la vidéo}

Le premier acte, en amont des séances filmées, est lié à la recherche des spécificités des notions à enseigner visées dont nous avons des traces dans les vidéos. C'est ce que nous appelons la recherche $\mathrm{du}$ « relief » sur ces notions. Ce relief, spécifique de chaque notion, comprend la caractérisation mathématique ${ }^{18}$ des notions qui interviennent, leur "fonction ", le spectre des problèmes qui peuvent y être attachés, mais aussi leur insertion dans les programmes scolaires et dans les acquis antérieurs supposés des élèves, ainsi que le listage des difficultés d'apprentissage déjà répertoriées. Cette démarche rappelle la mise au point du champ conceptuel qui englobe la notion (Vergnaud, 1991). Elle amène 
à préciser le niveau de conceptualisation visé, en termes à la fois de disponibilité pour les élèves des objets et outils en jeu (Douady, 1986), sur un ensemble de tâches supposées caractéristiques, et de (ré)organisation des connaissances anciennes et nouvelles.

Le deuxième acte consiste à reconstituer le scénario, choisi par l'enseignant, dans lequel s'insèrent les séances analysées, c'est-à-dire l'ensemble ordonné des cours et exercices correspondants ainsi que les contrôles et le travail prévu à la maison. Ces éléments de cours et ces énoncés d'exercices sont recueillis a posteriori, voire reconstitués, avec des indications grossières de gestion. Cela permet une première appréciation globale de ce qui a été proposé aux élèves : mise en évidence des dynamiques entre cours et exercices, de l'introduction choisie ${ }^{19}$, précision sur la variété des tâches mathématiques en référence au relief et au niveau de conceptualisation visé. Les mises en fonctionnement attendues des connaissances résultent d'une conversion par le chercheur des tâches analysées a priori en activités. Elles renseignent ainsi sur le travail possible des élèves, sur les dynamiques entre ancien et nouveau (notamment les introductions), entre sens et technique (gammes et/ou exercices complexes), entre signes et sens (rôle $\mathrm{du}$ formalisme) et plus localement sur les mises en fonctionnement attendues sur chaque tâche. Mais il manque encore des analyses de ce qui s'est passé dans les classes par-delà les contenus proposés, pour apprécier les activités mathématiques possibles des élèves.

\section{Avec la vidéo}

Ensuite, il s'agit d'accéder par des analyses de vidéo bien choisies, à ce que l'enseignant développe en classe en vue d'une mise en perspective de certaines caractéristiques observées de ses pratiques et des activités des élèves, rapportées ensuite au scénario $^{20}$. En s'appuyant sur les premières analyses a priori, on produit de nouvelles analyses des tâches précises proposées aux élèves. Puis, on effectue des analyses plus ou moins détaillées des déroulements effectifs, tels que la vidéo les dévoile, ce qui donnent accès aux activités possibles des élèves par comparaison avec les analyses a priori : elles sont inférées à partir des analyses a posteriori des séances. D'autres déterminants, plus globaux, sont alors convoqués pour préciser encore et interpréter les choix des enseignants et en dégager des alternatives éventuelles. Selon les recherches, on complète les analyses par des études de productions d'élèves, des analyses fines des discours, ou par des entretiens avec les enseignants.

À l'issue de ce type de recherches, les résultats obtenus conduisent par exemple à délimiter, sur un certain nombre de contenus, les marges de manœuvre individuelles des enseignants, au sein des contraintes, aussi bien institutionnelles (qui induisent par exemple un respect des programmes, très présent et assez analogue d'un enseignant à l'autre) que liées au public (des régularités particulières traversent l'enseignement dans les établissements regroupant des élèves défavorisés) ou aux personnalités en présence.

\section{Exemples de recherches menées dans ce cadre}

Par exemple la vidéo analysée ci-dessous a déjà servi, d'abord dans le cadre de comparaisons avec d'autres vidéos. L'entretien de ce professeur, après visionnement de la séance, a permis d'avoir une idée de son avis sur des modifications, renforcé par l'avis analogue de 6 autres enseignants placés dans les mêmes conditions (Beziaud, Dumortier, Robert, Vandebrouck, 2003).

Une première comparaison avec une vidéo du même enseignant en $4^{\mathrm{e}}$, sur une séance de géométrie analogue (par rapport au cours, en l'occurrence le théorème de Pythagore), a permis d'introduire l'hypothèse de la stabilité des pratiques d'un même enseignant en régime de croisière, la composante médiative des pratiques d'un enseignant étant constitutive de cette stabilité constatée, plus que les autres composantes (Robert, 2007, Chappet-Pariès, Robert et Rogalski, 2008). Cependant le grain de l'étude langagière de l'enseignant varie d'une recherche à l'autre, l'introduction de fonctions langagières et l'étude de certains marqueurs dans le discours de l'enseignant amenant à préciser et à confirmer la stabilité de gestion.

Une deuxième comparaison avec une vidéo d'un autre enseignant sur un exercice assez analogue en troisième d'un établissement regroupant des élèves défavorisés (Chappet-Pariès, 2007) a amené à mieux appréhender certains choix de gestion de ces enseignants soucieux de ne pas réduire leur enseigne- 
ment sous prétexte de difficultés des élèves - on a pu dégager que le temps de recherche individuel est plus long, surtout moins orienté dès le début, les aides sont plus procédurales encore mais tardives, les phases collectives semblent vécues comme plus difficiles à organiser.

Une troisième comparaison avec une vidéo d'un autre enseignant sur un exercice analogue (en quatrième) a amené à repérer des variables interenseignants - en matière de travail autonome des élèves (et du moment de le faire intervenir), d'aides, de repérage et d'appui sur ce que font les élèves.

\section{Analyses de vidéos en formation de formateurs}

On comprend sans peine comment ces résultats issus de la recherche peuvent nourrir le travail des formateurs en accroissant leurs connaissances sur le milieu professionnel qui va notamment absorber les débutants. Mais il ne saurait être question de reproduire ce travail effectué en recherche dans une formation de formateurs car les enjeux et les objectifs sont trop éloignés et la temporalité n'est pas du tout la même (cf. section suivante). En particulier, l'usage des vidéos est très différent et nous exposons en section III un exemple où il sera question d'une même vidéo utilisée en recherche et en formation de formateurs.

Dans la formation de formateurs dont il est ici question, les analyses de vidéo interviennent souvent, à plusieurs moments de la formation, mais, à chaque fois, de manière ponctuelle, isolée, et avec un statut variable au fur et à mesure de l'année. Elles servent dans un premier temps à donner du sens et à commencer à élaborer des réponses - souvent sous forme d'alternatives - à des questionnements collectifs des participants, initialisés par le chercheur-formateur, sur les pratiques en classe qui sont visionnées et décodées à partir des activités attendues des élèves. Cela conduit les participants à adopter une certaine posture d'analyse et non de jugement du travail de l'enseignant en relation avec ce qu'il provoque chez les élèves, en se mettant en quelque sorte " à sa place " sans avoir à faire les choix ni à en assumer les conséquences. Dans le même temps les participants, grâce au dispositif et aux outils d'analyse que le formateur a petit à petit mutualisés, souvent transposés des outils de recherche, se détachent quelque peu de la vidéo. Ils apprécient ainsi, de manière dépersonnalisée, les pratiques de l'enseignant du point de vue des tâches mathématiques qu'elles recèlent et des activités des élèves qu'elles ont pu provoquer. Les premières vidéos sont apportées par le formateur, à partir d'un stock dont il dispose. Les vidéos suivantes sont tournées dans les classes des participants et ce sont eux qui en analysent en séance collective, chacun à son tour et après préparation, un extrait. Il est à la charge du formateur, à chaque fois, d'accompagner et d'enrichir les inévitables remontées à des questions plus globales, associées à des déterminants des pratiques des enseignants, les programmes, la composition des classes, les diversités des représentations individuelles... Ce processus est décrit pour des formations d'enseignants conçues dans le même esprit dans un article sur ce type de séances (Chappet-Pariès et Robert, 2011).

Dans un deuxième temps, ces analyses de vidéo sont scrutées du point de vue de ce qu'elles peuvent apporter en formation et elles peuvent même être intégrées à des dispositifs de formation ${ }^{21}$, produits à la fin de l'année de formation.

\section{Points communs et différences dans les analyses de vidéos entre recherche et formation de formateurs}

Nous avons choisi d'expliciter quelques éléments qui nous semblent importants, avant de développer l'exemple. Le format d'analyses de vidéos dans les deux types d'activités est largement différent tout en ayant des points communs, puisque les analyses en formation sont inspirées par les recherches et que toutes s'inscrivent dans le même cadre théorique.

\section{Format d'analyse, documents complémentaires et exploitation}

Il y a une démarche commune aux analyses en recherche et en formation quand il s'agit d'analyser des moments d'exercices : c'est la mise en regard d'une analyse a priori des énoncés (analyse de la tâche) et des analyses de déroulement, de manière à comparer les activités attendues des élèves et ce qui peut se passer compte tenu du déroulement organisé par l'enseignant, même si les analyses a priori en formations sont plus rapides que celles en recherche. 
Ce qui peut être intéressant à cet égard est la détection de ce qui est transposé à la formation des outils du chercheur, en tenant compte de la progressivité de ce «transfert». Nous y reviendrons en conclusion.

Les consignes données en séance de formation sont toujours analogues : avant tout visionnement il est demandé d'analyser la tâche, en détectant les connaissances à mettre en fonctionnement et les adaptations attendues; puis on visionne un extrait, avec la consigne de mettre en regard les activités attendues des élèves et ce qui se passe ; un petit temps est laissé pour y réfléchir ; une mise en commun permet la mutualisation des analyses et la discussion; le formateur reprend la main à la fin. En recherche, il n'y a pas de consigne, mais une méthodologie de recherche. Si cette méthodologie peut reprendre les étapes précédentes, la différence essentielle concerne la maîtrise par le chercheur qui peut à tout moment la modifier pour s'adapter à son objet d'étude.

Les documents qui accompagnent le travail sont de plusieurs types, et là aussi très différents selon l'utilisation. Dans les deux cas on a à disposition les documents spécifiques à la séance, donnés par l'enseignant aux élèves ou propres à sa préparation. Cependant, si les questionnaires ou entretiens avec l'enseignant filmé sont nécessaires à la recherche, ils sont transcrits par écrit, ils ne constituent qu'un complément au visionnement en formation, complément dont on peut parfois se dispenser et qui est toujours oral (ou lu).

De plus, ces compléments au visionnement dans la formation sont, le cas échéant, toujours donnés après visionnement. En revanche, en recherche, de la même manière que l'étude du scénario et du relief précède les analyses de vidéo, tous les documents correspondants sont à disposition des chercheurs, dès la première écoute.

En formation, c'est en général après la discussion amorcée par le visionnement, souvent nourrie, autour des tâches, des activités des élèves et de celles de l'enseignant, voire des choix possibles (alternatives) et des tensions rencontrées que l'on "remonte» sous l'impulsion du formateur, à des questions plus générales qui peuvent se poser. Ces interventions du formateur permettent une exploitation spécifique qui n'a pas d'équivalent en recherche.

En recherche, les exploitations spécifiques d'une vidéo sans équivalent en formation sont nombreuses.
Nous renvoyons à la section $\mathrm{V}$ pour quelques exemples.

\section{Une temporalité différente pour les analyses, des questionnements spécifiques}

Une chronologie de la séance filmée est élaborée et est à disposition que ce soit en recherche ou en formation de formateurs (voir la section III) mais elle n'a pas tout à fait la même fonction. En formation de formateurs, comme on ne visionne tout au plus que $20 \mathrm{~min}$, il s'agit essentiellement d'avoir une idée globale de la séance, pour savoir comment le morceau visionné s'insère dans la séance, sans chercher à aller plus loin. En recherche, il s'agit également d'avoir une vision globale mais plus pour se repérer, se représenter l'enchaînement des épisodes pour notamment identifier d'éventuelles influences.

En ce qui concerne l'inscription temporelle des visionnements, il y a tout de suite une première différence dans les utilisations, très importante : dans la formation, on démarre très vite le visionnement de chaque vidéo après avoir donné quelques éléments de contextes et avoir fait faire une rapide analyse $a$ priori. Puis, on passe à l'analyse de l'extrait choisi de la vidéo qui est limitée en temps - le temps de la séance. Les questions concernent comme nous l'avons rappelé ci-dessus le devenir des activités des élèves attendues. Il n'y a pas plus d'une séance consacrée à chaque vidéo, on change de vidéo à chaque fois, que ce soit au début avec les vidéos du stock du formateur ou ensuite avec les vidéos des participants - le choix des extraits est fait par le formateur puis par les participants sur leurs propres vidéos. Autrement dit c'est à partir d'une (seule) vidéo qu'on enclenche la réflexion sur le scénario dont elle représente une séance. Cela interdit notamment une mise en relation directe avec les effets de la séance sur les acquisitions des élèves; cela s'inscrit en revanche tout à fait dans notre objectif de mise en ouvre d'outils d'analyse portant en eux les appréciations que nous voulons faire travailler : richesse et robustesse des tâches proposées.

La formation s'appuie sur cette diversité d'analyses et sur le temps long (plusieurs mois) qui est consacré à ce type de séances sur des thèmes mathématiques et à des niveaux scolaires variés, avec la partie " travaux pratiques » où chacun analyse sa propre classe, - cela permet d'avoir rencontré 
suffisamment de contenus différents, de déroulements variés et de discussions sur les alternatives et les tensions et d'installer le vocabulaire spécifique commun « emprunté » aux recherches.

En recherche a contrario, l'analyse de la séance, qui est souvent étudiée entièrement, est précédée comme nous l'avons déjà écrit ci-dessus par l'étude du relief et du scénario et elle est souvent complétée par un ou deux autres films du même enseignant, voire des productions d'élèves, voire, là encore, un ou des entretiens avec l'enseignant concerné, voire des vidéos permettant des comparaisons avec d'autres enseignants. De plus, très souvent, l'analyse nécessite plusieurs passages de la vidéo, voire même une étude plus ou moins fine de la transcription (réalisée par le chercheur). Un important travail de reconstitution plus global s'engage s'appuyant, entre autres, sur les données recueillies grâce à la vidéo, adapté à la problématique particulière dans laquelle s'inscrivent les visionnements. En outre, la durée d'analyse d'une vidéo n'est, en recherche, pas du tout limitée.

Ainsi le temps passé sur une vidéo n'est pas du tout le même en recherche et en formation. Mais au-delà de l'utilisation, la durée de vie des vidéos est également très différente en recherche et en formation. En formation, une vidéo peut très vite devenir obsolète du simple fait des changements curriculaires, même si le phénomène en jeu dans la vidéo est toujours d'actualité. Quel intérêt y aurait-il pour la formation de proposer une vidéo sur les triangles semblables en seconde enregistrée il y a une dizaine d'années ? Il semble en effet bien plus productif de proposer une autre vidéo, ancienne ou nouvelle, sur une notion du programme en vigueur. En revanche, en recherche, ces vidéos conservent tout leur intérêt. Elles permettent même de nouvelles études prenant en compte l'évolution dans le temps des systèmes éducatifs, les réformes.

\section{L'élève}

Revenons enfin sur l'absence en formation, qui peut sembler étonnante dans un premier temps, d'observations plus poussées d'élèves ainsi que de la question des effets d'une séance. Plusieurs expériences avec deux caméras, une centrée sur les élèves et l'autre sur l'enseignant ont été tentées et abandonnées. Cela nécessitait un observateur, voire un matériel lourd et troublait le déroulement. De plus il était difficile de synchroniser les deux visionnements. Les moyens technologiques actuels et d'autres expériences laissent penser que cela devient accessible - cependant l'exploitation en formation reste à élaborer, dans la mesure où cela modifierait le projet, qui nous semble, fondateur de la démarche, de travailler comme l'enseignant. Quant à la question des effets sur les acquisitions, il est évidemment illusoire de se limiter à une séance pour commencer à les apprécier. Cela différencie complètement nos analyses en formation de celles de Tiberghien et Malkoun (2007) qui travaillent au contraire sur la différenciation des pratiques en relation avec les acquisitions des élèves. Dans notre démarche, c'est une réflexion sur la palette des possibles pour l'enseignant, guidé par les apprentissages des élèves, qui est visée. Les dimensions dont nous pensons qu'elles peuvent influencer les apprentissages, par l'intermédiaire des activités des élèves, servent à construire les outils d'analyse et à apprécier les choix des enseignants : ainsi les tâches précises et leur ensemble, et la nature du travail des élèves ainsi que des accompagnements de l'enseignant.

En recherche, bien entendu tout dépend du sujet d'étude. En particulier une focalisation sur le travail d'un élève ou d'un groupe d'élèves est possible avec une caméra spécifiquement placée pour suivre le travail des élèves.

\section{UN EXEMPLE : DES ÉLÉMENTS PRÉALABLES D'ANALYSE COMMUNS EN RECHERCHE ET EN FORMATION}

La vidéo que nous allons prendre comme exemple générique a déjà été étudiée dans plusieurs articles, ce qui a permis de dégager son utilité en recherche (cf. ci-dessus). On a vu par exemple que des indicateurs plus ou moins précis ont été introduits selon les problématiques en jeu. Il s'agit d'une séance d'exercices sur le théorème de Thalès en troisième, dans un collège favorisé, au début des années 2000. Lors du film, c'est le programme précédent, datant de 2009, qui était en vigueur ${ }^{22}$. C'est le deuxième exercice de la séance qui est étudié, le premier, qui occupe le premier quart d'heure, correspond à la correction d'un exercice donné à chercher à la maison, mettant en fonctionnement la réciproque du théorème de Thalès. Une partie de la transcription de cet épisode figure en annexe. 
Voici l'énoncé que l'enseignante a préparé et qu'elle écrit au tableau :

EFG est un triangle tel que $E F=5 E G=7 F G=9(e n ~ c m)$

$\mathrm{M} \in[\mathrm{EF}]$ et on pose $\mathrm{EM}=x$

$\mathrm{N} \in[\mathrm{EG}]$ et tel que $(\mathrm{MN}) / /(\mathrm{FG})$

1) Exprimer EN et $\mathrm{MN}$ en fonction de $x$

2) Calculer $x$ afin que le périmètre du trapèze MNGF soit égal à 19,8 cm

Nous allons présenter des analyses de cet énoncé avant de travailler sur l'extrait du film qui correspond au début de l'exercice.

Une première analyse de la tâche (pour la recherche et en formation)

Cette analyse permet de lister les tâches et soustâches ${ }^{23}$ que peuvent accomplir des élèves de troisième pour résoudre cet exercice, compte tenu des programmes en vigueur et de l'état d'avancement du cours de la classe. Nous précisons les connaissances à mettre en fonctionnement et les adaptations attendues. Ce découpage en sous-tâches sert de trame pour l'analyse de la vidéo et à établir la chronologie (cf. ci-dessous).

\section{Liste des tâches}

- T1 Faire une figure. Cette première étape n'est pas indiquée explicitement dans l'énoncé. Il se pose alors la question du type de figure à réaliser : un tracé à main levée ou instrumenté (règle seule ou avec un autre instrument), en vraie grandeur ou non.

- T2 Reconnaître qu'il faut utiliser le théorème de Thalès dans la figure donnée et l'utiliser en adaptant l'énoncé du théorème donné en quatrième. Il faut en effet remplacer la longueur EM par $x$ ce qui correspond au passage d'un travail dans le cadre géométrique à un travail dans le cadre algébrique.

- T3 Faire une transformation algébrique sur des quotients qui font intervenir des nombres et des lettres, ceci deux fois de suite, de manière indépendante (pour obtenir EN et $\mathrm{MN}$ ). Il est à noter ici que ce travail sur les quotients dépend de la manière d'écrire les rapports dans le théorème de Thalès : l'application directe du cours donne l'égalité $5 / x=7 / \mathrm{EN}$ alors que le théorème de Thalès permet également une écriture de l'égalité $x / 5=E N / 7$ qui est plus simple à gérer pour obtenir EN en fonction de $x$ mais qui demande une adaptation dans l'utilisation du théorème.

- T4 Exprimer le périmètre d'un trapèze, dont la définition est à retrouver (connaissance ancienne supposée disponible), par une expression algébrique dépendant des résultats précédents et demandant d'ajouter NG et MF (intermédiaires). On retrouve le changement de cadres déjà signalé.

- T5 Mettre en forme et résoudre une équation d'inconnue $x$ qui se ramène au type $c=a x+b$, où $a, b$ et $c$ sont des décimaux qui s'écrivent, selon le registre utilisé, soit avec un chiffre après la virgule soit (pour $a$ et $b$ seulement) avec 5 pour dénominateur.

- T6 Vérifier que la solution est géométriquement acceptable, ce qui reste cependant implicite, notamment parce que la question porte sur $x$ et pas sur M.

Les étapes précédentes sont à peu près indiquées dans l'énoncé, à l'exclusion de la première et de la dernière et aux intermédiaires près; il n'y a pas d'indépendance complète entre les questions mais il n'y a ni conjecture préalable ni intermédiaire à introduire : le travail peut commencer rapidement.

\section{Quelques commentaires sur ces tâches}

Sur T2, l'utilisation du théorème de Thalès n'est pas "simple », c'est-à-dire que les élèves n'ont pas seulement à remplacer dans une expression de leur cours des données générales par des données issues du contexte. On peut penser que le fait que les lettres de l'exercice (E,F,G,M,N) ne soient sans doute pas celles du cours, ne pose plus de problème dans cette classe, alors que cela peut encore être une source de difficultés ailleurs. La figure de l'exercice est la même que celle du cours : il n'y a pas de tracé supplémentaire et on peut supposer que les élèves vont orienter le triangle comme dans le cours. En revanche le fait de remplacer une longueur EM par $x$ peut être une difficulté, ne serait-ce que vu la nouveauté de cette démarche (il s'agit de la première utilisation du théorème de Thalès avec « $x »$ ).

Sur T4, la définition du périmètre du trapèze est supposée mobilisable - ici la seule connaissance à mobiliser est celle du périmètre d'un quadrilatère 
convexe : il se trouve que la figure indiquée est un trapèze, mais cela ne sert pas. Les calculs algébriques nécessaires font intervenir des fractions non numériques dans lesquelles $x$ intervient plutôt comme un nombre généralisé (inconnu), remplaçant une longueur variable, et pour résoudre une équation du premier degré où $x$, cette fois-ci variable, est l'inconnue, même si les élèves n'ont pas besoin de l'identifier comme telle tout de suite; cette équation est à établir à partir des résultats précédents, avec un travail de mise en forme, la réduction des termes contenant $x$; puis il faut la résoudre. Le retour à la géométrie, pour tester la validité du résultat dans le contexte de l'exercice, n'est, répétons-le, pas du tout indiqué, ni même rappelé.

Tout ce qui précède est à peu près commun à la recherche et à la formation. Plutôt du côté de la recherche, on peut signaler les deux points suivants qui seront repris en section $\mathrm{V}$.

Sur T1, on peut signaler, mais sans obligation, en formation, des ambiguités qui peuvent intervenir dans certaines recherches. La figure n'est pas donnée tout à fait dans l'ordre de la construction puisque l'on signale que $\mathrm{N}$ est sur le côté [EG] avant de dire que les droites $(\mathrm{MN})$ et $(\mathrm{FG})$ sont parallèles. Est-ce que cela gêne des élèves ? Est-ce que l'enseignant en tient compte ? En outre, le statut de la figure peut être discuté. À partir de l'énoncé, on peut penser que la figure ne doit servir que comme support à l'utilisation du théorème de Thalès, donc plutôt en Géométrie II (Houdement et Kuzniak, 1999), mais il est fort possible qu'un nombre important d'élèves choisissent un statut plutôt du type Géométrie I. Une figure en vraie grandeur ou à main levée et le choix des instruments de tracé sont des indicateurs du statut de la figure et peut conduire à des interventions adaptées.

Sur T3, aucune précision n'est apportée sur l'écriture des nombres à utiliser, décimaux ou fractions. À ce propos, on peut remarquer que le théorème de Thalès oriente davantage vers le registre des fractions.

\section{Pour l'étude du déroulement - la chronologie (établie par le chercheur-formateur)}

Comme annoncé précédemment, une chronologie est donnée, en formation et en recherche. Elle est établie par le chercheur à partir d'un premier vision-

\begin{tabular}{|c|c|c|c|c|}
\hline $\begin{array}{l}\text { Tâche analysée } \\
\text { a priori }\end{array}$ & Nature de l'épisode & $\begin{array}{l}\text { durée de } \\
\text { l'épisode }\end{array}$ & $\begin{array}{l}\text { Nature et forme du travail pres- } \\
\text { crit aux élèves }\end{array}$ & $\begin{array}{l}\text { Côté enseignant (en gras : ce qui est ajouté } \\
\text { aux sous-tâches) }\end{array}$ \\
\hline $\mathrm{Tl}$ & $\begin{array}{l}\text { Lecture de l'énoncé } \\
\text { et construction de } \\
\text { la figure }\end{array}$ & $2 \cdot 20$ & $\begin{array}{l}\text { Écriture de l'énoncé } \\
\text { Faire la figure }\end{array}$ & $\begin{array}{l}\text { Écrit l'énoncé, dessine la figure } \\
\text { rapidement }\end{array}$ \\
\hline $\mathrm{T} 2$ & $\begin{array}{l}\text { Première ques- } \\
\text { tion : quelle } \\
\text { stratégie? }\end{array}$ & 535 & $\begin{array}{l}\text { Recherche collective de la straté- } \\
\text { gie pour la première question, } \\
\text { imposée immédiatement }\end{array}$ & $\begin{array}{l}\text { Jeu de questions, réponses et reprises - } \\
\text { réfléchir aux hypothèses et à la conclu- } \\
\text { sion, à ce que c'est que x, à une stratégie } \\
\text { L'utilisation du théorème de Thalès, } \\
\text { suggérée par un élève est confirmée }\end{array}$ \\
\hline T2-T3 & $\begin{array}{l}\text { Première ques- } \\
\text { tion : l'application } \\
\text { du théorème }\end{array}$ & 125 & $\begin{array}{l}\text { Recherche individuelle de la } \\
\text { solution (utilisant le théorème de } \\
\text { Thalès) }\end{array}$ & L'enseignant circule dans les rangs \\
\hline $\mathrm{T} 2$ & $\begin{array}{l}\text { Première ques- } \\
\text { tion : début de } \\
\text { la correction } \\
\text { (l'application du } \\
\text { théorème) }\end{array}$ & $2^{\prime} 10$ & $\begin{array}{l}\text { Correction de la première partie } \\
\text { de la première question par } \\
\text { un élève désigné pour aller au } \\
\text { tableau }\end{array}$ & $\begin{array}{l}\text { L'élève interrogé écrit au tableau en partie } \\
\text { sous la dictée. } \\
\text { Le professeur donne une suite de sous- } \\
\text { tâches à remplir - autour de l'utilisation } \\
\text { du théorème de Thalès et de la rédaction }\end{array}$ \\
\hline T3 & $\begin{array}{l}\text { Le calcul } \\
\text { algébrique }\end{array}$ & $40 "$ & $\begin{array}{l}\text { Reprise } \\
\text { Recherche individuelle }\end{array}$ & $\begin{array}{l}\text { Interruption après écriture des rapports, } \\
\text { interventions sur ' } x \text { ' }\end{array}$ \\
\hline T3 & $\begin{array}{l}\text { Première } \\
\text { question : fin } \\
\text { de la correction } \\
\text { (l'expression en } \\
\text { fonction de } \mathrm{x} \text { ) } \\
\end{array}$ & $2 \times 40$ & $\begin{array}{l}\text { Correction de la fin de la } \\
\text { première question par un autre } \\
\text { élève envoyé au tableau }\end{array}$ & $\begin{array}{l}\text { Découpage très précis - l'élève remplit des } \\
\text { «trous » } \\
\text { Discussion sur x puis sur l'écriture de } 7 x / 5\end{array}$ \\
\hline $\mathrm{T} 4 \ldots$ & $\begin{array}{l}\text { Deuxième } \\
\text { question (straté- } \\
\text { gie et recherche } \\
\text { individuelle) }\end{array}$ & $\begin{array}{l}2 ' 30 \\
+2\end{array}$ & $\begin{array}{l}\text { Deuxième question : straté- } \\
\text { gie collective - Recherche } \\
\text { individuelle }\end{array}$ & $\begin{array}{l}\text {... cet épisode et les suivants ne sont pas } \\
\text { analysés ici }\end{array}$ \\
\hline
\end{tabular}


nement, ponctué par les tâches listées ci-dessus, que l'on suit à la trace. Les épisodes, ou phases, sont ainsi repérés en référence aux activités attendues sur chaque Ti (colonne tâches). Il se peut qu'il y ait des ajouts ${ }^{24}$ comme de nouvelles tâches qui se repèrent ainsi directement par un vide dans la colonne tâche. Le découpage est ainsi établi de manière universelle tout en étant susceptible de varier à chaque exercice.

Cette chronologie, peut évidemment être simplifiée ou, essentiellement en recherche, complétée. Elle prend à peu près cette forme à laquelle on peut ajouter la durée cumulée depuis le début (en formation on ne propose qu'un des deux repères temporels : durée depuis le début ou durée de chaque épisode). Elle est donnée toute faite en formation de formateurs, en indiquant la manière dont elle a été établie - cela permet de situer dans l'ensemble les extraits visionnés (souvent de l'ordre de 15 à 10 minutes) et cela facilite l'unique visionnement proposé.

\section{L'UTILISATION DE CETTE VIDÉO EN FORMATION DE FORMATEURS}

C'est la première vidéo étudiée en formation de formateurs, après une séance consacrée à l'analyse des tâches et au repérage des connaissances à mettre en fonctionnement. Ainsi ont été développées les catégories utiles, connaissances anciennes, disponibles, adaptations des connaissances attendues dans les mises en fonctionnement (reconnaissances, introduction d'intermédiaires, découpages et étapes, mélanges de cadres ou registres ou points de vue, recours à des questions antérieures, choix...).

\section{Objectif et dispositif}

On pourrait intituler la séance où on analyse cette vidéo: "l'analyse des tâches c'est bien mais cela ne suffit pas!». Le travail sur cette vidéo (et les suivantes) sert en effet à sensibiliser les participants à l'importance du déroulement, à introduire les indicateurs utilisés pour inférer les activités possibles des élèves (a minima, a maxima) et de ce fait conduit à mettre en évidence la nécessité d'étudier ensemble, et pas séparément, tâches et déroulements ; ce visionnement amorce aussi les questions susceptibles d'être « remontées».
Le dispositif ${ }^{25}$ précis de cette séance (et de toutes les séances d'analyse de vidéo) est le suivant :

a) L'analyse individuelle des tâches de l'exercice du film est demandée et est suivi d'une mise en commun des analyses de chacun aboutissant à peu de choses près à l'analyse a priori ci-dessus (liste de connaissances et adaptations écrites au tableau).

b) Une chronologie simplifiée est présentée rapidement pour donner une idée de la séance entière dont on va visionner une partie.

c) Le visionnement (les 15 premières minutes de l'exercice) est proposé. On se restreint à la première question. Il n'y a pas d'autre visionnement, on ne donne pas la transcription - il $y$ a là un choix de formation ${ }^{26}$.

d) La mise en regard collective de l'analyse $a$ priori et de ce qui s'est passé (quelles activités des élèves) est organisée. En regard des tâches prévues (écrites au tableau), on étudie ce qui s'est passé dans la classe et on écrit ce que les participants proposent. On constate rapidement qu'il y a des différences dans les activités possibles selon les élèves, ce qui amène à distinguer les activités a minima, celles qui ne semblent s'enclencher qu'après toutes les interventions de l'enseignant sur le sujet et les autres, a maxima, qui peuvent même précéder le discours de l'enseignant. On fait constater alors qu'on a bien besoin d'une telle prise en compte du déroulement.

Une analyse collective de mise en regard de l'analyse a priori et du déroulement : quelles activités des élèves?

Reprenons sur la première question les soustâches prévues et ce qui peut être analysé, en décrivant rapidement ${ }^{27}$ ce qui résulte de la mise en regard des tâches prévues écrites au tableau et des déroulements tels que la vidéo les donne à voir, en séance de formation.

Cet « exercice » de recherche des décalages entre les deux donne lieu à des phrases souvent négatives - il ne faut pas le lire comme une succession de critiques mais bien comme un apprentissage du décryptage entre l'attendu et le réel (possiblement !), avec toute la richesse correspondante. Le formateur note les constats des participants en face des éléments de l'analyse a priori qui a été consignée au tableau 
dans ses grandes lignes, il reprend et reformule éventuellement les interventions.

\section{Constats des participants}

Les premiers constats portent sur le tracé de la figure : les participants remarquent ${ }^{28}$ qu'il est indiqué tout de suite par l'enseignant, ne laissant pas cette initiative à ceux des élèves qui n'auraient pas commencé très vite, et la discussion sur « vraie grandeur ou pas » est abordée également dans la classe, peut-être avant que tous les élèves se soient posé la question - ce qu'en dit l'enseignant laisse libres les élèves mais indique tout de même que ce n'est pas la peine de respecter les dimensions. Cela semble permettre à tous les élèves de tracer une figure, assez rapidement. Le professeur lui-même ne fait pas ensuite la figure à l'échelle et n'utilise que la règle, sans les graduations.

La reconnaissance nécessaire de l'utilisation du théorème de Thalès est provoquée par la question explicite immédiate de l'enseignant sur la stratégie lignes 39 à 46 sur la transcription en annexe ${ }^{29}$. Cela n'est pas laissé à l'initiative des élèves qui n'ont pas encore démarré ; on peut aussi souligner qu'avant même de lancer les élèves sur cette recherche intermédiaire, l'enseignant leur propose une sous-tâche non prévue : préciser les hypothèses et les conclusions de l'exercice (lignes 11 à 20). Cela se fait de manière un peu formelle, systématique, sans que ne soit explicitée la relation éventuelle de cette recherche avec une piste de démonstration. Même chose avec $x$ : avant même que le problème ne se pose, l'enseignant interroge sur ce $x$, reprenant les réponses des élèves et les arrangeant jusqu'à obtenir la réponse attendue ( $x$ est une variable) et complétant même par l'intervalle de définition de $x$, non demandé - lignes 25-38.

Revenons au théorème : les participants peuvent constater que son utilisation est reconnue par certains élèves qui répondent à la question du choix de stratégie - elle est confirmée et justifiée par l'enseignant (lignes 44-46), permettant aux autres élèves qui n'auraient pas encore trouvé de bénéficier d'une forme d'aide procédurale (utiliser le théorème de Thalès); tous les élèves sont donc prêts à cette utilisation, pour laquelle l'enseignant laisse un petit temps de recherche individuelle (tâche isolée si ce n'est simple, en tout cas sans reconnaissance).
Cette partie de la résolution est ensuite corrigée par un élève envoyé au tableau, qui commence par proposer la solution puis écrit la référence au théorème et les rapports mais sous un contrôle très strict de l'enseignant, souligné par les participants. Le format de l'écriture est une sorte de modèle de rédaction, d'emblée, "comme au contrôle » et l'enseignant rappelle au passage la norme qu'elle demande d'utiliser quand on cite le théorème mis en fonctionnement (lignes 50-51). Il y a amalgame dans cette correction entre ce qui pourrait être une reprise de la phase de recherche et la rédaction finale attendue. L'enseignant ne cherche pas à reprendre des difficultés ou erreurs d'élèves, il y a peu d'aides constructives explicites si ce n'est le renvoi à des tâches déjà bien connues.

Inattendu (lignes 68-73) : l'enseignant interrompt l'élève au tableau alors qu'il était en train de remplacer les longueurs par leurs mesures, dont $x$; l'enseignant ne s'en est pas aperçu tout de suite, il était en train de répondre à un autre élève. Quelques secondes sont laissées aux élèves pour qu'ils exploitent les égalités de rapport obtenues. On peut penser que, dans la mesure où la difficulté est justement là, l'enseignant isole le calcul algébrique qui pose problème.

\section{Qu'est-ce qui sert comme indicateurs pour mettre en regard tâches et déroulement?}

Le travail en formation se base sur un découpage selon les tâches listées auparavant par l'analyse $a$ priori (conversion des énoncés en mises en fonctionnement attendues des connaissances) - et on intercale ce que l'enseignant introduit.

Les questionnements (directs ou indirects) de l'enseignant, l'orientation au démarrage (prise en main, recherche de stratégie par exemple), le découpage du travail des élèves indiqué, notamment pendant la correction, les aides à fonction procédurale (donnant une méthode à suivre) ou constructive (s'appuyant sur le travail fait pour généraliser, justifier...), interviennent dans ces analyses mais aussi la forme et la nature du travail, les durées, les modes de correction et leur lien avec le travail des élèves, les bilans également en relation avec le travail des élèves.

Ces indicateurs sont suggérés par les théories de l'apprentissage que nous avons adoptées - ce qui « importe » pour comprendre les activités des élèves tient à la fois à leur travail individuel et collectif, à 
leur autonomie pendant ce travail, et à l'exploitation qui en est faite par l'enseignant à partir d'un repérage plus ou moins explicite du travail des élèves (faisant intervenir l'efficacité supposée de l'appui sur des connaissances des élèves " presque-là », ce que modélise la Zone Proximale de Développement).

\section{A-t-on « appris " quelque chose sur les activités des élèves? En avait-on besoin?}

La comparaison des tâches analysées a priori et des activités possibles appréhendées à partir du visionnement amène à constater qu'il y a des différences importantes. Par exemple beaucoup d'initiatives éventuelles attendues ne sont pas laissées à tous les élèves - réduisant peut-être la part d'adaptations potentielles pour certains, moins rapides, tout en les enrôlant de manière plus certaine que s'ils étaient laissés à eux-mêmes. Tracer la figure, utiliser le théorème adéquat, en particulier, sont rapidement indiqués par l'enseignant. Certains petits commentaires de l'enseignant, de type constructif, accompagnent les indications données - « alors bien sûr premièrement vous faites une figure » (ligne 8), et plus loin, «car évidemment on a des droites ... parallèles » (lignes 44-46). Est-ce entendu, est-ce suffisant pour que les élèves qui n'y avaient pas pensé le fassent la prochaine fois?

Par ailleurs, les premières interventions de l'enseignante, ajoutées et non prévues dans nos analyses de tâches, ne sont pas amorcées par le travail des élèves - elles sont de type " anticipation » en relation avec des difficultés qui vont se présenter comme "la seule nouveauté est $x$ » et reprises au moment de la rencontre de la difficulté (lignes 23, 73, 97) ou de type " mise en place d'habitudes ", sans lien direct avec des questions d'élèves, comme «écrire les hypothèses et la conclusion »(lignes 11 et suivantes). On peut se demander, compte tenu de ce décalage entre les tâches et les commentaires, ce que ces interventions produisent chez les élèves qui ne se sont pas encore posé les questions correspondantes.

En revanche, remplacer par $x$ la longueur EM dans le rapport obtenu par application du théorème, a une place spéciale : tous les élèves, même ceux qui n'en étaient pas là, ont un petit temps spécifique pour faire juste cette sous-tâche, certes préparée, mais il leur reste à faire le remplacement. Ainsi, au regard de l'analyse des tâches, l'adaptation essentielle attendue est bien à faire par les élèves mais de manière isolée pour certains, il y a une certaine séquentialisation (imprévue) des activités, provoquée par le fait de couper la correction de la première question en deux, en renvoyant à sa place le premier élève juste avant la correction de la deuxième partie.

Si on considère les activités possibles des élèves, ce qui est notre préoccupation, il se confirme ainsi qu'on en sait davantage après visionnement qu'avant et qu'on a des pistes de questionnement. On sait ainsi sur quoi les activités des élèves ont été hétérogènes, avec des activités a maxima et a minima observables : notamment sur la recherche de stratégie. Il se confirme ainsi que les élèves plus lents ne sont pas tous associés à ce qui pourrait contribuer à construire de la disponibilité (peut-on faire autrement ?). On sait que l'enseignant a introduit des sous-tâches implicites, imprévues, voire complémentaires : faire la figure (n'est-ce pas un réflexe à faire acquérir ?), respecter un format de travail systématique pour le repérage des hypothèses et de la conclusion (dans quelle mesure cela aide les élèves ?), chercher une stratégie avant de se lancer (cela présuppose-t-il ou contribue-t-il à élaborer une vue globale ?), couper le travail d'application du théorème du travail de traitement algébrique qui en découle. Reste à réfléchir systématiquement à ce qui pourrait changer dans un tel déroulement - c'est l'objet du paragraphe suivant.

\section{Alternatives et remontées}

Une part importante du travail pendant la séance de formation, à l'issue des analyses précédentes, consiste à réfléchir aux alternatives. Cette réflexion n'est pas menée pour l'enseignant filmé mais en toute généralité. Il y a selon les séances des variations, dans la mesure où le formateur amorce ses interventions par celles des participants. Nous présentons ici une sorte de moyenne de ce qui peut être travaillé de cette façon.

Notons d'abord que l'enseignant interrogé sur cette séance, qui a été interviewé après le film (le questionnaire correspondant étant distribué aux participants), reconnaît qu'il envisage peu d'alternatives. Son projet est de "mélanger » algèbre et géométrie, c'est bien le cas, et en termes de gestion, il a mis au point un optimum, un équilibre entre ce qu'il peut déléguer à ces élèves (de collège) et le maintien d'une attention de tous les élèves de la 
classe, enrôlement et recopie sur le cahier compris. En particulier le modèle donné au moment de la correction sert à « rattraper» les élèves qui n'auraient pas suivi et qui auront quand même de bonnes traces pour travailler chez eux.

Le travail sur les alternatives est donc plus général, et dépasse cette classe-là.

\section{Sur le même exercice, des alternatives de gestion, des alternatives d'énoncés}

Beaucoup d'énoncés sont proposés en vrac par les participants, en voici quelques-uns.

Selon la classe, on pourrait ne pas aider les élèves au démarrage : c'est-à-dire leur déléguer « l'installation $»$ du travail. On pourrait aussi ne pas découper en deux étapes l'écriture du théorème de Thalès et l'expression algébrique demandée. On pourrait enfin faire travailler les élèves en petits groupes 10 minutes d'abord... Dans tous les cas, il s'agit de diminuer les aides de l'enseignant ou de les déplacer et de modifier le découpage en sous-tâches donné en séance.

Certes, mais les participants insistent sur le fait que cela peut renforcer les différences entre élèves, car dès qu'on envisage un travail plus long en autonomie, on peut évoquer un risque de décrochage pour les plus faibles n'ayant rien à faire. Dans le type de gestion de la séance filmée, tout le monde sait quoi faire et le fait apparemment, quitte à ne pas bien recoller les morceaux.

Certains changements d'énoncés sont aussi proposés, plus découpés (en commençant par un exemple, du style EM = 2), ou moins découpés (exprimer en fonction de $x$ le périmètre du trapèze et calculer $x$ afin qu'il soit égal à 19,8). On retrouve la même utilisation du théorème de Thalès a priori, mais plus cachée (provoque-ton un travail plus important sur la disponibilité du théorème ?). Dans cette version, les élèves ont à introduire eux-mêmes les longueurs à calculer et notamment l'intermédiaire EN pour obtenir NG.

Mais on peut se demander si une gestion analogue à celle de la séance analysée serait bien adaptée aux énoncés moins découpés : en effet les contraintes d'une gestion dialoguée, guidant suffisamment les élèves, amèneraient peut-être à l'introduction de trop de sous-tâches intermédiaires en contradiction avec l'ouverture de l'énoncé.
Ainsi apparaît une idée fondamentale : on ne peut pas séparer l'analyse du déroulement de celle de la tâche. Tel énoncé ne se conçoit pas avec tel déroulement, pourtant adéquat à un énoncé différent du même exercice.

\section{Introduction d'un logiciel}

Parmi les logiciels actuellement utilisés au collège, un Logiciel de Géométrie Dynamique (LGD) et le tableur sont tous deux envisageables pour le type d'exercice envisagé ici. Ces deux types d'outil ne sont toutefois pas identiques ni sur les activités mathématiques qu'ils proposent aux élèves, ni sur leurs utilisations effectives en classe (Kuzniak, Parzysz \& Vivier, 2013).

L'utilisation d'un LGD est très efficace pour résoudre l'exercice sur le périmètre du trapèze une fois la figure réalisée. Car contrairement à une figure papier/crayon, on peut rapidement faire calculer le périmètre du trapèze pour un point $\mathrm{M}$ particulier puis déplacer le point $\mathrm{M}$ pour ajuster ce périmètre pour obtenir 19,8 puis lire (ou faire mesurer) la longueur EM. Cette utilisation est simple et la figure est utilisée dans une perspective de type Géométrie I (Houdement et Kuzniak, 1999). La question qu'il faut alors se poser est la suivante : quelles sont les mathématiques en jeu ? De fait, l'activité mathématique est relativement pauvre (cela pourrait même être proposé dès la classe de sixième) surtout si l'objectif est l'utilisation du théorème de Thalès. Toutefois, en modifiant les données numériques pour que la solution $x$ ne soit pas un décimal simple, la question de l'exactitude de la solution pourrait susciter des discussions et des développements qui mèneraient sur le chemin du théorème de Thalès on reconnaît l'importance des variables didactiques dans les alternatives.

Le tableur permet également une exploration numérique du périmètre pour l'ajuster à 19,8. Mais à la différence d'un LGD, il est nécessaire de faire calculer les longueurs en fonction de la position du point M. La différence avec l'exercice est qu'il n'est pas nécessaire de nommer la mesure EM par la lettre $x$ car seule une mesure suffit : par exemple, en fixant $\mathrm{EM}=3,2$ (par exemple en mesurant sur une figure papier/crayon) on peut trouver que EN est égale à $7 / 5$ de cette mesure ce qui se traduit dans le tableur, si EM est en $\mathrm{A} 2$ et $\mathrm{EN}$ en $\mathrm{A} 3$, on entre dans cette dernière 
cellule $"=7 / 5 * \mathrm{~A} 2 »$ (on multiplie par $7 / 5$ la valeur de EM qui se trouve en A2, le A2 de la formule peut être obtenu par un simple clic sur la valeur de EM qui s'y trouve effectivement). Évidemment, cela suppose une utilisation du théorème de Thalès. La différence essentielle avec l'exercice proposé concerne l'usage de l'algèbre dont on peut alors se dispenser si ce n'est pas un des objectifs de la séance (c'était un des objectifs du professeur). Mais cet usage du tableur nécessite en amont une prise en main de cet outil avec notamment son utilisation pour la recherche d'une solution à un problème pouvant se modéliser par une équation.

\section{Retour à des questions plus globales}

Des questions peuvent émerger dès qu'on réfléchit à ces alternatives - du côté du scénario, du travail sur le théorème de Thalès, et des difficultés de ce chapitre - quelle place de cet exercice dans le scénario ? Ces questions appellent ce que nous qualifions de « remontées »-introduites par le formateur - on les aborde de plus en plus au fur et à mesure de l'année dans ces séances de formation, nous n'en donnons ici qu'un bref aperçu - cette première séance de la formation ne faisant qu'amorcer ce processus.

Du côté des mathématiques, un élément important qui structure les réflexions globales sur l'enseignement d'une notion donnée tient à ce que nous appelons la détermination du type de la notion (Robert, 1998). C'est une démarche pour apprécier, pour une classe donnée, dans un programme donné, la distance entre les connaissances déjà introduites aux élèves et la nouvelle connaissance à enseigner et, de ce fait, pour élaborer une introduction appropriée. Cette distance est appréhendée au travers de plusieurs caractères de la notion, en relation avec les connaissances déjà rencontrées, généralisateur, formalisateur, unificateur et simplificateur. Selon le degré de formalisation par exemple, une notion pourra être considérée ou non comme extension de notions déjà introduites, avec ou sans accidents d'ailleurs. Élaborer un « bon problème » d'introduction sera évidemment fonction de cette « distance » - s'il y a un saut trop grand, il sera difficile de trouver un problème abordable pour les élèves et les amenant à adapter leurs connaissances déjà là pour le résoudre. Cela a amené à distinguer deux types de notions. D'une part les notions « réponse à un problème » qui se laissent bien aborder par un problème mais uniquement du côté des questions à poser, les élèves n'ayant pas les moyens d'approcher ce qui va permettre de résoudre. D'autre part les notions s'exprimant dans un nouveau formalisme, généralisant et unifiant des connaissances précédents, souvent en les simplifiant (mais pour l'expert !) et dont l'introduction aux élèves est difficile.

Un autre élément structurant les scénarios tient aux dynamiques qu'ils recèlent - entre ancien et nouveau, sens et technique, outils et objets, signes et sens, et à la quantité et à la variété des tâches introduites (cf. adaptations).

Dans le cas qui nous occupe, le théorème de Thalès est introduit en quatrième dans une version allégée, qui peut être présentée (au mieux) comme une réponse à un problème. En troisième on peut s'appuyer sur ce qui a été fait précédemment et introduire des adaptations nombreuses - notamment, comme ici, en mélangeant algèbre et géométrie, soulevant en particulier des questions d'ordre dans les scénarios. Ces questions ne sont abordées que si les participants eux-mêmes les soulèvent. Des exemples en sont donnés dans Chappet-Pariès \& Robert (2011).

\section{Bilan et suite}

Les objectifs de la séance vidéo sont en partie atteints !

L'importance du déroulement pour apprécier les activités des élèves a été dégagée - il y a des activités imprévues, d'autres réduites, des décalages entre les tâches et les interventions, et des différences entre les activités des élèves. Les participants ont été confrontés à l'utilité d'étudier ce déroulement en relation étroite avec l'analyse de tâches. Des outils ont été donnés (grâce à des indicateurs permettant de faire le lien entre tâches et activités possibles des élèves). Cela a amené à questionner les choix (plus globaux) correspondants.

Les séances suivantes de la formation de formateurs reprennent ces objectifs en les approfondissant - mise au travail plus systématique des indicateurs, remontée aux questions liées aux choix des enseignants et aux contraintes correspondantes, aux mathématiques enseignées et aux programmes imposés, aux difficultés des élèves et aux différents types d'établissements. L'objectif est que la prise de conscience provoquée par les premières études, suite 
aux questionnements et à la mise en évidence des alternatives, pourra être enrichie par la palette des possibles qui s'élabore au fur et à mesure.

\section{EXEMPLES DE COMPLÉMENTS DU POINT DE VUE DU CHERCHEUR}

Pour préciser ce que nous avons annoncé plus haut, nous donnons quelques exemples de ce qui pourrait être ajouté au sein d'une recherche utilisant cette vidéo (cela dépend bien entendu de la problématique). Le plus souvent on étudie en même temps la transcription, et cela s'accompagne souvent d'un minutage de la séance (ajouté dans ce paragraphe). Ce travail peut compléter les analyses ou conduire à de nouvelles questions grâce à une étude plus fine du discours de l'enseignant à partir de la transcription, des phrases précises de l'enseignant voire des fonctions du langage ou des marqueurs langagiers (Pariès, Robert et Rogalski, 2008). Il se peut qu'une discussion en séance de formation amène à utiliser un de ces compléments.

\section{Sur la figure}

Ici l'intervention du professeur relative à la phrase "Alors bien sûr premièrement vous faites une figure » (ligne 8, 1'42) est évidemment évoquée en formation de formateurs mais seulement dans la perspective des activités des élèves. En recherche, et sans revenir sur les ambiguités discutées précédemment, plusieurs questions peuvent en découler et la transcription verbale est essentielle pour s'y référer et pour identifier les mots importants. Peut-être y a-t-il une volonté du professeur d'aider rapidement tous les élèves dans la tâche proposée, mais cela ne peut suffire à expliquer les mots employés et notamment « bien sûr » et " premièrement » qui ont des visées plus globales. Le professeur fait-il simplement référence à une habitude de classe ? Essaie-t-il d'instaurer un contrat didactique? Mais alors, dans ce dernier cas, les élèves ont-ils les moyens de repérer des éléments déclencheurs qui font que, pour une tâche donnée, il faille faire premièrement une figure ?

De fait, après une digression sur le repérage des hypothèses et conclusions de l'exercice, le professeur précise (ligne 22, 4’45) : «Bon alors ceci dit vous faites une figure, donc je répète que vous pouvez la faire à main levée, enfin à main levée ou avec votre règle, mais sans respecter les dimensions, si ça vous aide de respecter les dimensions allez-y. » Les données numériques n'excluent pas en effet une construction en vraie grandeur, sauf pour le point $\mathrm{M}$, voire un mesurage de contrôle. On reconnaît là une variable didactique implicite de l'énoncé (le choix des longueurs des côtés). Se pose aussi le choix de la technique de tracé de la parallèle : à main levée ou instrumentée (règle seule ou avec un autre instrument) ainsi que le choix de la position de M.

L'enseignant donne bien le statut de la figure visée même s'il laisse une possibilité aux élèves de faire autrement. On peut dire ici que le professeur lève les ambiguités sur le type de figure finalement à produire. Le visionnement plus spécifique du moment où le professeur effectue la construction (lignes 50 et 51, 7’30) est en adéquation avec la précision précédente. On remarque que le seul instrument utilisée est la règle - pour tracer les segments -, les graduations de la règle ne sont pas utilisées et ainsi la figure réalisée au tableau n'est pas semblable à la figure de l'énoncé (de plus la taille relative des segments n'est pas conservée puisque [FG] est le plus petit côté sur son dessin ; les élèves ne le relèvent pas). Les points et les mesures sont écrits ensuite comme si le professeur réalisait une figure générique du théorème de Thalès qu'il fallait ensuite instancier avec les données de l'exercice - ainsi, la droite (MN) est tracée, avec la règle et à vue, avant de nommer les points. Le placement de $\mathrm{M}$ et $\mathrm{N}$ se fait ensuite, ce qui est différent de l'énoncé et nécessite une reformulation qui n'est peut-être pas si évidente que cela. En effet, ce n'est plus M qui est variable mais la parallèle à $(F G)$. On comprend tout de même l'intérêt pratique pour le tracé de la figure car on a alors qu'une seule contrainte à respecter au lieu de deux dans l'énoncé initial. La distance entre la figure du professeur et celle que pourrait effectuer de lui-même un élève peut paraître importante pour beaucoup d'entre eux.

Le statut de la figure géométrique est un des enjeux de l'enseignement de la géométrie au collège ce que Houdement et Kuzniak (1999) interprètent comme un changement de paradigmes. Mais ce statut dépend notamment de l'énoncé et on ne donne généralement que peu d'éléments aux élèves pour qu'ils puissent d'eux-mêmes comprendre le type de figure attendu. Quelles incidences une intervention et une clarification rapide, comme celles de cet enseignant, ont-elles? 


\section{Séquentialisation du travail des élèves dans différents cadres}

En ce qui concerne la gestion de la séance donnée à voir par la vidéo, reprenons l'imprévu de la correction de la question 1. Le professeur interrompt l'élève au tableau en disant "qu'est-ce que tu fais? " (ligne 68,10 '50) au moment où l'élève était en train de remplacer les valeurs des longueurs dans les égalités du théorème de Thalès. En formation, l'essentiel est de montrer le découpage de l'activité proposée aux élèves qui permet d'isoler les difficultés (utilisation du théorème puis basculement dans l'algèbre). En recherche on peut aller un peu plus loin car il s'agit également d'une séquentialisation des cadres en jeu. Il semble que le professeur désire ainsi séparer le travail dans les deux cadres (géométrique - et aussi grandeurs et mesures - puis algébrique). Si elle ne prend pas en charge le changement de cadre, elle l'accompagne fortement. Bien entendu cela peut être dit en formation de formateurs, ne serait-ce que pour préciser la difficulté en jeu. Mais en recherche ce sont les questions qui se posent à la suite qui sont porteuses. Souvent, les exercices proposés aux élèves (celui en question ici, mais aussi ceux des manuels) ne laissent pas à la charge des élèves l'introduction d'un cadre ou d'un registre (ici, on donne le $« x »$ dans l'énoncé). Mais ici, on va plus loin car l'enseignant précise l'endroit où intervient le changement de cadre.

Les jeux de cadres (Douady, 1986) sont essentiels pour le travail et l'apprentissage des mathématiques. Ici, le professeur semble retirer, au moins à l'élève au tableau, la responsabilité de changer de cadres. Est-ce souvent/toujours le cas ? Est-ce un trait de ce professeur ou bien une constante institutionnelle?

\section{Sur la preuve}

Enfin, le passage sur les hypothèses et conclusions (lignes 11 et suivantes, de 2'20 à 4'35) «à entourer » est perçue de manière un peu anecdotique en formation, mais en recherche on pourrait développer ce point en discutant de la forme des démonstrations qui peut prendre le pas sur le sens.

Plus spécifiquement, pour la vidéo en question, alors que le professeur semble très à cheval sur la rigueur et la forme de la démonstration, on note lors de la correction au tableau par une élève (ligne 65,
9'30) que l'hypothèse sur l'ordre des points, pourtant précisé dans l'exercice précédent celui étudié et qui est rappelé à l'oral (cf. ligne 50), disparaît dans la rédaction comme le montre la transcription suivante du tableau :

Dans le triangle EFG M€(EF) et $\mathrm{N} \epsilon(\mathrm{EG})(\mathrm{MN}) / /$ $(F G)$ d'après le théorème de Thalès EM/EF $=\mathrm{EN} /$ $\mathrm{EG}=\mathrm{MN} / \mathrm{FG}$

Ces deux points posent des questions sur le sens que peuvent donner les élèves aux démonstrations: des blocs entourés " hypothèses » et " conclusion * auxquels il faut ajouter quelque chose - renforcé parfois avec d'autres blocs proposés et à intercaler et une rigueur flottante.

\section{Sur les registres numériques}

Les coefficients $7 / 5$ et $9 / 5$ qui interviennent peuvent être ou non transformés en décimal. Cela d'autant plus que dans la question 2, le décimal 19,8 est donné. Éventuellement il y a donc à faire un choix dans l'écriture des coefficients de $x$ intervenant dans les expressions de EN et MN, entre d'une part 9/5 et $7 / 5$ et d'autre part 1,8 et 1,4 (ces derniers nécessitant un calcul supplémentaire). La difficulté de ce calcul, une conversion de registres (Duval, 1995), est différente selon que les élèves ont à disposition une calculatrice ou non. Le calcul à la main demande soit de poser la division (9/5) soit de chercher une fraction décimale égale $(9 / 5=18 / 10)$. Il est à noter que des élèves font effectivement ce calcul mais on ne sait pas de quelle manière.

Le choix de $7 / 5 x$ ou de $1,4 x$ n'est pas neutre et se pose pour les élèves (ligne 112). L'écriture 7/5x met l'accent sur une interprétation en lien avec les grandeurs, où $x$ est une longueur dont on prend les $7 / 5$, un fractionnement de grandeur, alors que l'écriture 1,4x met plus l'accent sur la multiplication de deux nombres. Pourquoi l'enseignant favorise-t-il la première interprétation ? et dans ce cas, pourquoi avoir choisi 19,8 cm comme périmètre qui favorise, implicitement par le choix du registre, la deuxième interprétation? 


\section{CONCLUSION}

\section{Des questions anciennes et nouvelles}

\section{Quelles vidéos?}

La place de la vidéo dans nos dispositifs, au fond de la classe, se justifie par l'absence d'observateurs dans la classe pendant la captation - cela nous avait été demandé par les premiers apprentis-formateurs avec lesquels nous avons travaillé à ce type de projet. Il est en outre plus facile d'oublier une caméra placée au fond de la classe - donc hors champ visuel qu'avec un autre dispositif, avec ou sans observateur.

D'autres dispositifs peuvent être envisagés avec un contrat initial différent. La question du choix des vidéos reste aussi posée - sur quelles séances (quelle notion, séance d'exercices, de cours...), avec quels enseignants... L'utilisation de deux vidéos du même enseignant, ou deux vidéos d'enseignants différents sur un même énoncé, peut ajouter une certaine richesse.

L'expérience nous a montré qu'il se produit une certaine « naturalisation » de l'usage de la vidéo dans la durée - ce qui ne veut pas dire qu'il est facile de se voir exposé(e) aux yeux des autres, ni de présenter et discuter ses choix sereinement, en revenant aux mathématiques avant d'argumenter sur ses élèves.

Cela dit, la spécificité de la vidéo, au regard d'enregistrements audio par exemple, n'est pas encore dégagée complètement - cf. note 8 . C'est pour sa grande proximité aux pratiques effectives (vues et entendues) et pour sa relative simplicité d'utilisation qu'elle s'est imposée à nous.

\section{Retour sur la transposition entre recherches et formation}

Nous avons illustré dans les deux analyses de l'extrait de vidéo précédent quelques éléments que nous transposons systématiquement, à savoir les variables mises en jeu dans les analyses de tâches et de déroulement et le travail qui est fait pour décrire et discuter les choix. Cependant le complément spécifique « recherches» que nous avons joint au paragraphe précédent met bien sur la piste d'une vraie difficulté en termes de transposition, à savoir les limites de ce qui peut être transféré d'une utilisation à l'autre. En effet pour les formateurs, autant ce qui peut outiller le travail d'analyse et de critique, dans toutes circonstances, est appréciable, autant l'intérêt de ce qui apparaît encore particulier, isolé, sans ancrage dans des choix possibles, reste discutable. Ainsi les analyses très fines, qui débouchent souvent plus sur des questions que sur des régularités, n'alimententelles pas les formations.

\section{Des pistes à explorer}

Les questions de l'évaluation des formations restent toujours posées. Ce quadruple chantier, des formations, des formateurs, des effets sur les pratiques des enseignants formés et des effets de celles-ci sur les apprentissages de leurs élèves, ne peut s'aborder uniquement quantitativement mais, cela posé, il reste presque vierge !

Nonobstant, de nouvelles perspectives s'ouvrent, aussi bien en recherches qu'en formation : par exemple, à partir d'une réflexion sur la notion de « genre » introduite par Clot (2000), il semble important de caractériser des groupes professionnels dont les enseignants qui les constituent partagent plus ou moins certaines pratiques, éventuellement en partie à leur insu (Butlen et Robert, 2011). En particulier il reste certainement beaucoup d'éléments à repérer dans ce qui se joue en classe qui restent implicites, liés aux signes, aux habitudes très naturalisées des enseignants, éventuellement rendues encore plus « transparentes» par l'appartenance à un groupe professionnel qui en renforce l'usage non questionné.

\section{Une question ouverte : c'est quoi «la classe"?}

Plus localement, une question très ouverte tient à l'appréciation de la classe, cet ensemble d'élèves qui ne peut pourtant s'y réduire. Exploiter collectivement le travail des élèves nécessite par exemple un repérage de ce qui a été fait en vue d'une certaine mutualisation. Est-ce toujours possible? Dans quelle mesure cela intervient pour justifier certains choix instantanés de l'enseignant ? Plus généralement, la question des indicateurs à utiliser pour apprécier ce qui se joue en classe, en recherche et en formation, entre activités effectives et possibles, entre une séance et l'ensemble des séances sur le même thème, est fondamentale et encore ouverte. 


\section{Ici et ailleurs}

Les analyses de vidéo en recherche et en formation ont donné lieu à beaucoup d'articles depuis une dizaine d'années. Ce n'est pas le lieu de discuter les analyses en recherche. Nous n'avons pas la place pour faire une revue des recherches sur les utilisations en formation (pour une revue des utilisations de la vidéo en formation d'enseignants novices, quelle que soit la discipline cf. Gaudin \& Chaliès, 2012). Mais nous voulons d'abord souligner que c'est davantage les analyses de vidéo en formation d'enseignants qu'en formation de formateurs qui sont étudiées. Beaucoup d'articles décrivent des dispositifs particuliers, partageant des hypothèses, pas toujours explicites, mais assez proches nous semble-t-il, sur l'intérêt de faire travailler les enseignants, débutants ou non, à partir de films montrant des pratiques en classe. Souvent on donne des grilles d'observation à renseigner qui attirent l'attention sur des caractéristiques jugées importantes de ces pratiques, liées à la prise en compte des élèves, à la possibilité de repérer leur compréhension (la rendre « visible » ou non), à la possibilité d'élaborer des alternatives, y compris en termes de contenus (Santagata, 2011, Sherin, 2004). Il y a même des évaluations qui sont faites sur l'évolution de l'appréciation des vidéos entre le début et la fin de la formation - ce qui laisse quand même non renseignée la question du lien avec les pratiques (Stürmer, 2011).

Soulignons enfin le développement, dans d'autres pays, de contextes différents de ceux qui sont traditionnellement envisagés pour la formation $^{30}$ : recherches collaboratives, communautés de pratiques, « Lesson study »...

\section{Formateurs : un enjeu spécifique}

Notre approche de la formation met en jeu la possibilité d'enrichir les activités des enseignants, que ce soit à l'installation ou plus tard. Cet enrichissement est lié à la fois à une extension de la palette des possibles présentée et discutée en formation et aux modalités de celle-ci. Il doit permettre aux participants d'adopter une posture proche de celles des enseignants au travail et par là même de travailler sur le travail enseignant. Les séances correspondantes, « à l'envers », partant d'un travail sur des séances ou même des extraits, et permettant la remontée à des questions plus générales sont inhabituelles.
Cela amène à travailler des séances de formation à partir de séances de classe (vidéos notamment) qui demandent un travail très important mais très particulier du formateur. Il doit à la fois organiser les discussions, amenant questionnements et prises de conscience, grâce à la mutualisation d'un vocabulaire professionnel adapté " minimal », qu'il doit s'être approprié et contribuer à remonter à des éléments plus globaux que ce qui a été échangé - ce qui demande des connaissances élargies et une grande disponibilité.

Il ne s'agit pas de ramener à sa propre expérience ou à sa propre pratique ce qui est visionné et discuté mais bien d'accepter la diversité et de lui donner sa place dans le champ des possibles. Et cela demande des moyens de décrire le travail de l'enseignant, de l'apprécier sans le juger, d'avoir accès à la littérature pour mieux cerner les possibles sur l'enseignement d'une notion, et d'avoir à sa disposition des hypothèses sur les formations, suffisantes à élaborer des dispositifs de formation permettant de provoquer ces questionnements, prises de conscience et, au final, enrichissements de la palette des actions possibles en classe.

C'est ce qui justifie notre entreprise de formation de formateurs, longue et diplômante. Signalons que d'autres recherches donnent un rôle particulier et important aux formateurs, rejoignant globalement notre démarche, même si ce qui leur est dévolu est un peu différent (Coles, 2012).

Parmi les questions qui ne manquent pas de se poser, on peut soulever, en relation avec ce texte, celles du choix des vidéos à utiliser en formation (d'enseignants). Des conditions nécessaires président à toute utilisation : inscription dans un scénario cohérent, consistant - travail des couples énoncésxdéroulements, remontée à l'ensemble des énoncés et cours et aux autres déterminants. Cependant des variables sans doute importantes restent à discuter, par exemple le choix des vidéos, avec comme variables les sujets des séances ou les auteurs des vidéos (participants ou non), le choix des conditions de visionnement (durée, analyses préalables, exploitation...). En particulier, le (chercheur)-formateur ne joue pas le même rôle dans les deux types de vidéos. Avec les premières vidéos, choisies par lui, il dispose de ses analyses préalables avec un double regard de chercheur/formateur qui condense la transposition. En revanche, avec les vidéos des participants, les analyses se font en direct, 
elles ne sont pas préparées et le double regard, bien que toujours présent, est vraisemblablement moins important.

Entre recherche et formation de formateurs : des débats

Un certain nombre d'outils issus de la recherche sont importés dans cette formation, mais ils sont transposés puisqu'ils sont utilisés dans un autre contexte, et de ce fait peuvent apparaître un peu artificiels, peu justifiés, ou réduits. La définition de ce que nous avons transposé s'est faite petit à petit, pilotée par notre démarche d'allers-retours entre ce que les formateurs peuvent nous apprendre et ce que nous avons envie de leur apprendre. Cette démarche nécessite en effet le partage de catégories précises, de mots bien définis et elle préside finalement au choix des outils et des éléments théoriques à mutualiser.

Des débats nombreux alimentent cependant ce type de transposition. Des chercheurs suggèrent par exemple que c'est la théorie didactique dans son intégralité (et intégrité) qui doit être mise à portée des formateurs, voire des enseignants - c'est le cas pour la Théorie Anthropologique du Didactique. D’autres courants de recherche travaillent davantage dans la direction de recherches collaboratives, qui ne nécessitent pas de tels emprunts, aux chercheurs revenant l'interprétation didactique de ce qui est partagé.

Poser le problème à partir d'un exemple précis nous a semblé intéressant à ce titre, pour contribuer à préciser ce qu'il en est, dans un premier temps ! 


\section{Annexe \\ Transcription du travail sur la première question de l'exercice analysé}

Ce qui est dit par les élèves figure en italique, les numéros aident à se reporter.

1. Alors EFG est un triangle; l'énoncé va être court mais vous l'écrivez s'il vous plaît.

2. EFG est un triangle tel que, alors je vous donne ...

3. $\mathrm{EF}=5, \mathrm{EG}=7, \mathrm{FG}=9$ et l'unité est le centimètre. On va prendre un point $\mathrm{M}$ qui appartient au segment [EF] et on pose $E M=x$. N sera un point du segment EG qui sera tel que $(\mathrm{MN})$ va être parallèle à $(\mathrm{FG})$.

4. Non on va on va l'écrire comme ça. Je donne exprès l'énoncé sous forme très mathématique pour qu'on n'écrive pas uniquement les hypothèses et la conclusion parce que ça va être clairement écrit.

5. Alors premièrement exprimer donc EN et MN en fonction de $\mathrm{x}$

6. Question d'élève...C'est exprès Madame que vous faites?

7. Et deuxièmement pardon B. ? et calculer $\mathrm{x}$ afin que le périmètre eh bien du trapèze MNGF soit égal à 19,8 cm. Voilà.

8. Alors bien sûr premièrement vous faites une figure

9. Question d'élève... en vraie grandeur?

10. Eh bien c'est comme vous voulez, c'est comme vous voulez parce que on peut très bien faire ce problème sans faire grandeur nature donc c'est comme vous voulez. Si vous comprenez mieux la situation quand c'est grandeur nature eh bien vous le faites, là je pense que ça n'a pas tellement d'intérêt. Donc vous faites une, une figure, vous marquez les indications dessus.

11. Alors pour répondre à $\mathrm{R}$. tout à l'heure qui me disait ben est-ce qu'on écrit les hypothèses et est-ce qu'on écrit la conclusion, on va faire comme on fait assez souvent pour gagner du temps, dans le texte même, qu'est-ce que je vais faire R. ? En deux couleurs?

12. Où est-ce que je vois les hypothèses tout simplement?

13. Dans l'énoncé, c'est-à-dire les trois... premières lignes d'accord. Eh bien c'est ce que vous allez faire, on l'a déjà fait, hein ça nous gagne du temps. Voilà.

14. Alors on va marquer hypothèses,

15. Alors là c'est conclusion. Pour la première donc le but c'est clair, calculer EN et MN en fonction de x donc ça ça fait bien partie du travail à faire donc des conclusions, mais est-ce que c'est si simple dans la deuxième question ? Estce que c'est si simple, est-ce que je vais entourer tout par mon trait pour voir si c'est heu justement le but ou est ce qu'il y a une information qui serait donnée à la deuxième question?

16. A.?

17. Ben le périmètre de MNGF est égal à 19,8

18. Voilà. Et ça ça fait partie de quoi ?

19. Des hypothèses

19. Des hypothèses.

20. Alors vous allez faire attention, donc on va marquer ici conclusion, d'accord sauf qu'on va mettre du côté hypothèses le 19,8. Donc vous faites attention
21. Le 19,8 concerne le périmètre du trapèze. D'accord ? 22. Bon alors ceci dit vous faites une figure, donc je répète que vous pouvez la faire à main levée, enfin à main levée ou avec votre règle, mais sans respecter les dimensions, si ça vous aide de respecter les dimensions allez-y.

23. Donc la situation est assez banale, hein, vu tout ce que l'on a fait, quelle est la seule nouveauté Be. ?

24. Euh

25. x c'est-à-dire le point $\mathrm{M}$, qu'est-ce que tu dis du point $\mathrm{M}$ ?

26. Ben on ne connaît pas sa vraie place sur EG

27. On ne connaît pas sa vraie place sur le segment EG, heu autrement dit c'est un point?

28. Inconnu

29. inconnu, quel mot d'autre on pourrait,

30. dont on ne connaît pas la place,

31. dont on ne connaît pas la place, $\mathrm{M}$ ?

32. variable

33. variable, $x$ est une variable, le point $M$ varie et alors $x$ il va varier de quoi à quoi ?

34. De... ben de 0 à 7 .

35. De 0 à 7 , on va même pouvoir l'écrire dès le début, on nous le demande pas, hein, une fois on avait fait un problème où on le demandait, mais on écrit tout de suite que $\mathrm{x}$ est compris entre

0 et 7

36. 0 et 7 . D'accord?

37. 0 et 5

38. 0 et 5 ? J'ai pas fait attention à... M est sur EF et EF attention il fait 5 . Ah tu as inversé, tu fais attention. Tout le monde a eu le temps de faire sa figure? Ça va ?

39. Alors avant de démarrer, qui est-ce qui me dit comment on va procéder?

40. Le début me semble sans tellement de surprise. T.

41. En fait $\mathrm{M}$ appartient à $\mathrm{EF}, \mathrm{N}$ appartient à $\mathrm{EG}$

42. Attends tu me dis la grande idée, tu vas rédiger convenablement sur le cahier.

43. Ben on va dire qu'on applique le théorème de Thalès 44. Voilà on va appliquer le théorème de Thalès parce qu'on évidemment on a des droites

45. parallèles

46. Des droites parallèles. Alors la seule chose qui va être un petit peu différente de d'habitude, c'est que

47. Ce sera $x$

48. Y aura $x$. Bon alors vous démarrez, allez-y

49. On le met n'importe où alors.. faut qu'ils soient parallèles...

50. On met n'importe où le point $\mathrm{M}$ et MN est une parallèle à FG et maintenant on applique son théorème de Thalès en le rédigeant comme on l'a fait dans l'exercice de tout à l'heure. Pendant ce temps-là je fais la figure, ça va ?

51. Allez on y va A. ? On rédige et on rédige comme dans un contrôle. 


\section{Aline Robert et Laurent Vivier}

52. Tu voudrais bien allez rédiger le début s'il te plaît, on va rédiger jusque-là puis après on posera hein.. Alors

53. Madame,

54. Tu essaies d'écrire là, voilà bon tu parles en même temps.

55. Oui il y a une question?

56. Heu il faut mettre à l'extérieur de, le point M peut être en dehors du point $\mathrm{N}$

57. Alors dans l'exercice est ce que c'est ... précisé regarde bien, $\mathrm{M}$ appartient à quoi ?

58. Ah au segment.. ;

59. Voilà hein, dans l'exercice, tu as raison dans le cas général tu pourras avoir un point sur la droite.

On t'écoute Laura.

60. Dans le triangle EFG, oui, heu M appartient à (EF)

61. Oui

62. et $\mathrm{N}$ appartient à (EG). Oui, et $\mathrm{MN}$ est parallèle à $\mathrm{FG}$

63. Voilà, on a tout ça. On sait qu'on a tout ça. Donc par hypothèse on a tout ça.

64. D'après le théorème de Thalès,

65. D'après le théorème de Thalès alors je vous rappelle que quand on est en train de rédiger, d'appliquer le théorème de Thalès il n'y a plus de si alors. Quelques-uns, peu mais deux trois ont rédigé l'exercice sur feuille là avec si les droites sont parallèles alors : je vous rappelle que ça c'est l'énoncé général de cours et nous on est en train de l'appliquer. Donc on sait ou on ne sait pas que les droites sont parallèles. Donc on marque on a ou par hypothèse ou on sait que là elle a rien marqué du tout, mais heu on ne doit pas marquer si

66. Alors après que EM/EN est égal à EN/EG

67. Madame, On est obligé de remettre ... Machin machin c'est écrit juste au -dessus

68. Oui parce que là t'es en train de rédiger, hein. Heu dans un contrôle par exemple, le texte y t'est donné si tu veux, c'est pas toi qui est en train de, d'expliquer. Donc on doit toujours reprendre les phrases de l'énoncé. Qu'est-ce que tu fais là ?

69. Maintenant j'ai j'ai reporté les mesures

70. Je reporte les mesures, et il se trouve que pour EM tu as mis

71. $\mathrm{x}$

72. $\mathrm{x}$

73. EM c'est égal à 5, EN on le cherche EG c'est $7 \mathrm{MN}$ on le cherche aussi et FG c'est 9 ; bon alors là tu tu poses ta craie, voilà, et on attend un petit moment. Donc ça c'est vraiment la partie que on commence un peu à connaître par cour, la nouveauté c'est donc là qu'il y a x.

74. Alors la nouveauté vous faites attention c'est aussi quel est le verbe qui est marqué là $C$. ?

75. Exprimer

76. Exprimer et je n'ai pas mis

77. calculer.

78. Calculer. Ça veut dire quoi ?

79. Ça veut dire

80. Attends laisse Chloé

81. On ne pourra pas trouver une valeur exacte
82. Voilà on ne pourra pas trouver, on on trouvera une valeur, on trouvera une valeur

83. par rapport à $x$

84. par rapport à $\mathrm{x}$, en fonction de $\mathrm{x}$.

85. D'accord ? Alors ... il y a une nouveauté. Je vous laisse chercher deux minutes.

...

86. [Ça n'a pas d'importance si tu veux. Hein, puisque maintenant tu es en troisième, donc on peut que $M$ soit sur le segment ou sur la demi-droite ou sur la droite ça revient toujours au même.]

87. Alors on y va. On écrit EN en fonction de $\mathrm{x}$ et on écrit MN en fonction de $\mathrm{x}$.

88. Vous faites comme vous, comme d'habitude, oui tu laisses trente secondes aux autres, ton cahier...

89. On écrit tout comme d'habitude mais on a un nombre qui s'appelle $\mathrm{x}$. Fais comme d'habitude comme si x était 3 ou 7, tu fais comme d'habitude

90. Je comprends pas exprimer

91. Exprimer EN en fonction de $x$ ça veut dire qu'il te manque un nombre tu tu vois bien qu'il te manque un nombre pour calculer comme on faisait d'habitude,

92. Oui

93. D'accord ? tu vas le faire à l'aide de la lettre $\mathrm{x}$

94. Le x il peut valoir $1 ; 1,5 ; 3$, tu n'en sais rien, il s'appelle $\mathrm{x}$. Donc dans le calcul tu vas exprimer en fonction de $\mathrm{x}$.

95. FG Madame c'est heu

96. Pardon? A. L.

97. Donc la nouveauté c'est que justement il faut qu'on fasse un calcul en traînant entre guillemets $x$. Voilà il y en a qui $y$ sont arrivés. Y en a qui n'ont pas eu peur de $\mathrm{x}$ et qui sont même allés plus loin c'est très bien.

98. En troisième ce que tu as fait avant tu l'utilises....

99. Tu veux aller écrire au tableau s'il te plait ?

100. Alors tu ... chut, s'il vous plait. Donc vous faites attention parce que c'est là la nouveauté hein et c'est ça qui est intéressant. Alors on va donc faire l'expression de EN. Bon voilà. Alors tu sélectionnes déjà tes rapports (intéressants). ...

101. Tu te retournes s'il te plaît?

102. Si tu veux... Alors est-ce que ça va, Olivia d'accord? On a traité le, le x finalement comme un nombre courant. La seule chose c'est qu'on va peut-être écrire heu EM sous une autre forme on est un peu habitué à écrire comment?

103. Oui le $\mathrm{x}$ on le met devant?

104. Non voilà, donc on va marquer $7 / 5$

105. Oui $7 x$ sur 5 voilà, tu vas l'encadrer ça là puis on va le laisser comme ça je vais être un petit peu embêtante parce que je vais laisser les fractions. Je sais bien que ça peut être un nombre décimal mais pour la suite je veux que vous vous entraîniez parce que si c'était des tiers vous pourriez pas si vous aviez $7 / 3$. Alors tire un trait vertical peut-être, voilà. Et qu'est-ce que tu me fais maintenant? 106. On va calculer $\mathrm{MN}$ 
107. On va exprimer MN hein on peut pas dire calculer vraiment

...

108. Donc là on sélectionne eh bien le premier et le dernier rapport, alors tu nous dit $\mathrm{M}$.

109. Oui, oui ben je vais exprès de traiter avec les fractions Mais c'est pas faux, Madame

110. Ce n'est pas faux évidemment. Bien alors voilà la première question. Donc on a des réponses en fonction de $\mathrm{x}$. Je vous rappelle que $7 \mathrm{x}$ sur 5 alors ça peut s'écrire de différentes manières, y en a certains... levez le doigt qui ont écrit avec un nombre décimal, par exemple L.

111. Qu'est-ce que t'as écrit?

...112. 1,4 x c'est-à-dire que tu as fait 7 divisé par 5 d'accord et puis ben donc 1,4 x. Ça veut dire aussi que $7 \mathrm{x}$ sur 5 c'est 7 cinquièmes de $\mathrm{x}$. T'es d 'accord? Alors la première question est faite...

\section{NOTES}

1. Sans observateur donc. Il y a déjà eu et il y a de plus en plus d'autres utilisations, quelquefois même très sophistiquées (école Michelet à Talence, Clarke 2007).

2. Seuls des questionnaires ont été recueillis (cf. Robert, in actes colloque Cergy).

3. Horoks (2008), Chesnais (2009).

4. Cela distingue notre approche de la démarche anthropologique beaucoup plus universelle, mais moins liée aux sujets singuliers.

5. On retrouve l'idée d'invariant dans cette description en termes de disponibilité des connaissances.

6. Cela rend notre orientation de recherche complémentaire de celle de la Théorie des Situations Didactiques par exemple, qui s'intéresse à l'élaboration de conditions nécessaires à l'avancée du savoir dans la classe.

7. Nous parlons de choix plutôt que d'intentions, dans la mesure où nous analysons les décisions correspondantes en les rapportant à des possibles que nous dégageons.

8. Il y a d'autres accès possibles et nous n'engageons pas la discussion théorique sur la manière dont le film peut rendre compte de l'action (Sensevy, 2011).

9. Sans pour autant permettre d'apprécier directement les effets en termes d'apprentissage.

10. Les activités a minima sont les activités que les élèves semblent développer tardivement, après toutes les interventions de l'enseignant, alors que d'autres élèves ont déjà travaillé, sans attendre, ce que l'on attribue à des activités a maxima.

11. Nous faisons cette brève incursion en formation d'enseignants car la même démarche est reprise en formation de formateurs.

12. Ensemble ordonné des cours et exercices, y compris les évaluations, avec des prévisions grossières de gestion a priori.

13. Aucune recherche précise n'étaye encore ces hypothèses, admises.

14. Ce qui peut inquiéter, en termes de moyens de contrôle notamment. D'autres didacticiens estiment qu'il est nécessaire de former les formateurs à la didactique, évitant ainsi cet écueil, mais les éloignant dans un premier temps des pratiques ordinaires.

15. Quelquefois complétées par un travail sur la littérature professionnelle

16. L'étude portait sur les triangles semblables en seconde alors encore au programme.

17. L'étude portait sur la symétrie orthogonale en sixième. 18. Éventuellement avec une incursion dans l'Histoire. 
19. La qualité de l'introduction des notions est source de beaucoup de recherches en didactique, dans la mesure où elle porte un enjeu important en matière de « sens » pour les élèves.

20. Il y a d'autres possibilités de découpage (Tiberghien et Malkoun, 2007), ce n'est pas le lieu d'en discuter.

21. Il s'agit de projets de formation sur un thème précis pour un public ciblé.

22. On note peu de différence sur le thème en question entre l'ancien et le nouveau programme ce qui légitime son utilisation en formation. Ce point a été signalé aux participants à la formation qui n'ont nullement été gêné.

23. Les tâches sont référées aux connaissances mathématiques auxquelles les élèves peuvent faire appel et, le cas échéant, au contrat développé dans la classe.

24. Par exemple, lors de l'écriture de l'énoncé de l'exercice au tableau, l'enseignante précise à l'oral que l'unité est le $\mathrm{cm}$, et que l'énoncé est écrit avec le formalisme mathématique, probablement pour abréger. Les symboles $\epsilon$ et // sont respectivement lus « appartient » et « parallèles » (cf. la transcription en annexe, lignes 3 et 4).

25. On peut déjà constater que cette utilisation est tout à fait distincte de l'utilisation en recherche, quant à sa place dans l'activité des participants, à ses objectifs, et aux inférences qu'on en fait, même si les 4 phases suivantes sont communes, la phase d) étant cependant souvent plus détaillée, avec des visées plus précises, particulières, en recherche.

26. Cela augmenterait considérablement le temps à consacrer à une vidéo pour un gain peu évident. Les participants, ayant effectué une analyse a priori de la tâche, suivent attentivement la vidéo ce qui suffit largement à soulever la majeure partie des points importants (les autres points importants peuvent être apportés par le formateur). Du point de vue de la formation, cet unique visionnement est en phase avec le métier d'enseignant puisque l'on ne joue qu'une fois une séance avec une même classe.

27. Il n'y a pas eu de relevé systématique de ce déroulement - nous nous appuyons sur le fait qu'il a eu lieu plus de 10 fois, avec des déroulements très proches, dont nous retraçons ici les grandes lignes.

28. Dans la suite du texte on ne précise plus « les participants remarquent que » mais c'est sous-entendu partout, que ce soit un participant ou plusieurs qui soient à l'origine du commentaire.

29. Les participants à la formation n'ont pas la transcription, mais les lecteurs de cet article n'ont pas la vidéo !

30. Même si certains groupes qui travaillent dans les IREM s'en rapprochent.

\section{RÉFÉRENCES}

Altet, M., \& Donaro, B.J. (1985). Micro-enseignement et formation des enseignants. Revue Française de Pédagogie, 71, 63-65.

Ball, D.L., \& Bass, H. (2003). Toward a practice-based theory of mathematical knowledge for teaching. In B. Davis \& E. Simmt (Eds.), Proceedings of the 2002 Annual Meeting of the Canadian Mathematics Education Study Group, 3-14. Edmonton, AB : CMESG/GCEDM.

Béziaud, P., Dumortier, D., Robert, A., \& Vandebrouck, F. (2003). Questionnaire sur l'utilisation du tableau. Document pour la formation $n^{\circ} 1$, IREM Paris 7 .

Brousseau, G. (1998). Théorie des situations didactiques. Grenoble: La pensée sauvage.

Butlen, D., \& Robert, A. (2012, sous presse). Interroger la profession en didactique des mathématiques, un filtre pour apprécier les activités possibles des élèves et des enseignants - et interroger la didactique ! Actes de la $X V I^{e}$ école d'été de didactique des mathématiques, Carcassonne.

Chappet-Pariès, M. (2007). Enseigner les math en ZEP et ailleurs. Cahier de Didirem n ${ }^{\circ}$ 55, IREM Université Paris-Diderot.

Chappet-Pariès, M., Robert, A., \& Rogalski, J. (2008). Analyses de séances en classe et stabilité des pratiques d'enseignants de mathématiques expérimentés du second degré. Educational Studies in Mathematics, 68, 55-80.

Chappet-Pariès, M., \& Robert, A. (2011). Séances de formation d'enseignants de mathématiques (collège et lycée) utilisant des vidéos (exemples). Petit x, 86, 45-77.

Chesnais, A. (2009). L'enseignement de la symétrie axiale en sixième dans des contextes différents : les pratiques de deux enseignants et les activités des élèves. Thèse de doctorat, non publié, Université Paris 7-Diderot, Paris.

Chesné, J.-F. (2006). La formation des pratiques chez les enseignants du second degré : des passages obligés ? Mémoire de master 2 non publié, Université Paris 7, Paris.

Clarke, D. (2007). Facilitating reflection and action: The possible contribution of video to mathematics teacher education, Oberwolfach (Allemagne), Novembre 2007. En ligne : < http://extranet.edfac.unimelb.edu. au/DSME/lps/DC/>.

Clot, Y. (2000). Une Clinique de l'activité. Education permanente, 146.

Coles A. (2013). Using video for professionnal development : the role of the discussion facilitator. Journal of Mathematics teacher education. 16-3 165-184.

Crahay, M. (1989). Contraintes de situations et interactions maître-élève, changer sa façon d'enseigner, est-ce possible ? Revue française de pédagogie, 88, 67-94.

Douady, R. (1986). Jeux de cadres et dialectique outil-objet. Recherches en Didactique des Mathématiques, 7(2), 5-31.

Duval, R. (1995). Semiosis et pensée humaine. Berne : Peter Lang. 
Gaudin, C. \& Chaliès, S. (2012). Lutilisation de la vidéo dans la formation professionnelle des enseignants novices, Revue Française de Pédagogie, 178, 115-130.

Goigoux, R. (2007). Un modèle d'analyse de l'activité des enseignants. Education et didactique, 1(3), 47-70.

Horoks, J. (2008). Les triangles semblables en classe de seconde : de l'enseignement aux apprentissages. Recherches en didactique des mathématiques, 28(3), 379416.

Houdement, C., \& Kuzniak, A. (1999). Sur un cadre conceptuel inspiré de Gonseth et destiné à étudier l'enseignement de la géométrie en formation des maîtres. Educational Studies in Mathematics, 40(3), 283-312.

Kuzniak, A. (1994). Etude des stratégies de formation en mathématiques utilisées par les formateurs de maîtres au premier degré. Thèse de troisième cycle, non publié, Université Paris 7, Paris.

Kuzniak, A., Parzysz, B., \& Vivier, L. (2013). Solving problems in French curriculum and teachers' training. The Montana Mathematics Enthusiast Journal, Special Issue: International Perspectives on Problem Solving Research in Mathematics Education, Guest Edited by Manuel Santos-Trigo \& Luis Moreno-Armella, vol. 10, $1 \& 2$, 407-440.

Leplat, J. (1997). Regards sur l'activité en situation de travail. Paris : Presse Universitaire de France.

Masselot, P., \& Robert, A. (2007). Le rôle des organisateurs dans nos analyses didactiques de pratiques de professeurs enseignant les mathématiques. Recherche et Formation, 56, 15-31.

Robert, A. (1998). Outils d'analyses des contenus mathématiques à enseigner au lycée et à l'université. Recherches en Didactique des Mathématiques, 18(2), 139190.

Robert, A. (2001). Les recherches sur les pratiques des enseignants et les contraintes de l'exercice du métier d'enseignant. Recherches en Didactique des Mathématiques, 21(1.2), 57-80.

Robert, A. (2005). Des recherches sur les pratiques aux formations d'enseignants de mathématiques du second degré : un point de vue didactique. Annales de didactique et sciences cognitives, 10, 209-250.

Robert, A. (2007). Stabilité des pratiques des enseignants de mathématiques (second degré) : une hypothèse des inférences en formation. Recherches en didactique des mathématiques, 27(3), 271 - 312.

Robert, A. (2008). La double approche didactique et ergonomique pour l'analyse des pratiques d'enseignants de mathématiques et une méthodologie pour analyser les activités (possibles) des élèves en classe. In F. Vandebrouck (Éd.), La classe de mathématiques : activités des élèves et pratiques des enseignants (45-68). Toulouse : Octarès.

Robert, A. (2010). Formation professionnelle des enseignants du second degré. Repères-IREM, 80, 87-103.

Robert, A. (2012, sous presse). Des recherches en didactique des mathématiques aux formations d'enseignants - l'exemple d'une formation de formateurs d'enseignants du secondaire ; la question des effets. In M.L. Elalouf \& al. (Eds) Les didactiques en questions, un état des lieux (actes du colloque de Cergy, 2010). Bruxelles : de Boeck

Robert, A., \& Rogalski, J. (2002). Le système complexe et cohérent des pratiques des enseignants de mathématiques: une double approche. Revue canadienne de l'enseignement des sciences, des mathématiques et des technologies, 2(4), 505-528.

Robert, A., \& Rogalski, J. (2005). A cross-analysis of the mathematics teacher's activity. An example in a French 10th-grade class. Educationnal studies in mathematics, 59, 269-298.

Roditi, E. (2003). Régularité et variabilité des pratiques ordinaires d'enseignement. Le cas de la multiplication des nombres décimaux en sixième. Recherches en didactique des mathématiques, 23(2), 183-216.

Rogalski, J. (2003). Y a-t-il un pilote dans la classe ? Une analyse de l'activité de l'enseignant comme gestion d'un environnement dynamique ouvert. Recherches en didactique des mathématiques, 23(3), 343-388.

Rogalski, J. (2005). Piaget et Vygotski : apports croisés pour une approche développementale. In C. Castela \& C. Houdement Eds, Actes du séminaire national de didactique des mathématiques, 237-262.

Rogalski, J. (2008). Le cadre général de la théorie de l'activité. Une perspective de psychologie ergonomique. Des compléments sur les théories de l'activité et du développement, pour l'analyse des pratiques des enseignants et des apprentissages des élèves. In F. Vandebrouck (Éd.), La classe de mathématiques : activités des élèves et pratiques des enseignants (23-30 \& 429-459). Toulouse: Octarès.

Santagata R. (2011). Video and Teacher learning: a multidimensionnal framework for using and studying video as a Tool for Teacher learning, in Filmer en classe et après? Symposium international Cadivam, HEP, Lausanne, juin 2011.

Sensevy, G., Mercier, A. Schubauer-Leoni, M.L., \& Leutenegger, F. (2007). Agir ensemble: l'action didactique conjointe du professeur et de l'élève. Rennes: Presses Universitaires de Rennes.

Sensevy, G. (2011). le sens du savoir. Bruxelles : De Boeck.

Sherin M. (2004). New perspectives on the role of video in teacher education, in Brophy J. (Ed.) Advances in research on teaching, vol. 10 Using video in teacher education, pp. 1-27. Boston MA: Elsevier.

Shulman, L.S. (1986). Paradigms and research programs in the study of teaching. In M.C. Wittrock (Ed.), Handbook of research on teaching. New York: MacMillan.

Stürmer K. (2011). Observe - identifying professional vision as indicator of teacher's professional competencies, in Filmer en classe et après? Symposium international Cadivam, HEP, Lausanne, juin 2011.

Tiberghien, A., Malkoun, L. (2007). Différenciation des pratiques d'enseignement et acquisition des élèves du point de vue du savoir. Education et didactique, 1, 29-54. 
Valsiner, J. (1992). Vygotsky's sociohistorical psychology and its contemporary applications, by Carl Ratner (book review). Early Development and Parenting, 1, 5658.

Vandebrouck, F. (Ed.) (2008). La classe de mathématiques : activités d'élèves et pratiques d'enseignants. Toulouse : Octarès.

Vergnaud, G. (1991). La théorie des champs conceptuels, Recherches en didactique des mathématiques, 10(2.3), 133-170.

Vygotski, L. (1997). Pensée et langage. Paris: La dispute.

Une référence sur « Lesson study »:

http://www.noycefdn.org/documents/math/lessonstudy. pdf 